\title{
Modal analysis of a grand piano soundboard at successive manufacturing stages
}

\author{
Roberto Corradi, Stefano Miccoli, Giacomo Squicciarini ${ }^{1}$ \\ Politecnico di Milano, Department of Mechanical Engineering, Via G. La Masa 1, 20156 Milano, Italy
}

Paolo Fazioli

Fazioli Pianoforti, Via Ronche 47, 33077 Sacile, Italy

\begin{abstract}
Manuscript Info
[May 14, 2017] This is an author generated postprint of the article:

"R. Corradi, S. Miccoli, G. Squicciarini, P. Fazioli, Modal analysis of a grand piano soundboard at successive manufacturing stages, Applied Acoustics, Volume 125, October 2017, Pages 113-127, IssN 0003-682X, DOI 10.1016/j.apacoust.2017.04.010',
\end{abstract}

The version of record is available at https://doi.org/10.1016/j.apacoust.2017.04.010

(C)2017. This manuscript version is made available under the Creative Commons Attribution-NonCommercialNoDerivatives 4.0 International License. (๔) Br-Nc-ND

\section{Abstract}

This paper deals with the development and validation of a Finite Element numerical model of a grand piano soundboard. The most important details of the manufacturing process are reproduced in this model. In particular, by modelling the gluing of the ribs and the bridges to the board, the curved geometry (the crown) and the residual stresses induced in the soundboard are taken into account.

Experimental modal analysis results are presented at three stages of the manufacturing process of the same soundboard: (i) freely suspended soundboard before gluing the bridges, (ii) freely suspended soundboard including the two bridges, and (iii) soundboard attached to the piano frame. The experimental data at the first of these three stages are used to update the material properties of the Finite Element model, so as to minimise the difference between calculated and experimental vibration modes, in terms of both natural frequencies and mode shapes. The simulation of the other manufacturing stages is then performed without any additional tuning. Vibration modes obtained from experiments up to $400 \mathrm{~Hz}-450 \mathrm{~Hz}$ are compared with those obtained through the numerical model.

Point mobilities measured on the bridge at the different manufacturing stages are also shown and discussed. Above $200 \mathrm{~Hz}$, the mobilities measured on the bridge of the freely suspended soundboard and on the bridge of the soundboard fixed to the frame are very similar, thus showing that in this frequency range the effect of the soundboard local dynamic stiffness is predominant over boundary conditions. Finally, point mobility at the bridge is also calculated through the Finite Element model and compared to the experiments, with good agreement up to $4 \mathrm{kHz}$.

Keywords: piano soundboard, experimental modal analysis, bridge point mobility, numerical modelling, FEM

\section{Introduction}

In a piano the soundboard has the role of converting string vibration into sound. Strings and soundboard are connected through bridges and the soundboard itself is stiffened by a set of ribs running perpendicular to the main direction of the wood grain. A grand piano soundboard is a complex structure, whose

\footnotetext{
${ }^{1}$ Currently Institute of Sound and Vibration Research, University of Southampton, United Kingdom.
}

elaborate manufacturing process introduces subtle changes in its shape and stress state. From the origin of the scientific studies on pianos [1] it was found that even the most slight modification of the soundboard geometry can have a perceptible effect on the tone quality. Specifically it is believed that all the following soundboard characteristics need to be considered and opportunely designed: the board variable thickness ("diaphragmed" soundboard), the ribs section and spacing, the material properties, the residual curvature after ribs gluing ("crown"), the static 
deflection due to the vertical component string loads ("downbearing") with the consequent residual stresses, the bridge position, and the way the soundboard is glued onto the rim. At the purpose of correlating these characteristics with the final tone quality, piano manufacturers are looking for reliable methodologies capable of predicting the acoustic behaviour of their instruments under design modifications, thus limiting the need for building prototypes.

The literature on piano acoustics is vast and covers aspects from hammer-string contact to sound radiation. We will focus our attention on those studies specifically devoted to soundboard testing and modelling or those that include the soundboard as an important aspect of the research. Several examples exist in the literature dealing with vibration testing performed at the purpose of validating and updating numerical models or simply aimed at characterising the vibrational and acoustic behaviour of the structure.

Early measurements of point impedance measured at the bridge of an upright piano were first presented by Wogram in [2] and later reported also in [3]. The effect of gradually increasing string tension was analysed and shown to shift only the first few resonances by roughly $50 \mathrm{~Hz}$. The reliability of these measurements above $1 \mathrm{kHz}$ was later questioned and seems to be a consequence of the shaker set-up and not due to a high frequency behaviour of the soundboard [4]. Wogram also performed modal analysis on a grand piano soundboard and showed the mode shapes of the first eight modes. The natural frequency of the lowest mode was measured at $62 \mathrm{~Hz}$ while that of the eighth mode was $325 \mathrm{~Hz}$. Nakamura [5] investigated the mode shapes of an upright piano and showed mechanical impedance at various locations on the bridge. He found that clamped boundary conditions give a good representation of the actual behaviour of the soundboard. Also in this case impedance appear to be reliable only below $1 \mathrm{kHz}$; above this frequency results were affected by the resonances of the shaker and of the sensor. Suzuki [6] presented a modal analysis of a grand piano soundboard below $200 \mathrm{~Hz}$ and acoustic and vibration measurements up to $5.4 \mathrm{kHz}$ to estimate the soundboard radiated power and radiation resistance. Measurements were performed on a soundboard with rim but without strings and cast-iron frame. In this condition Suzuki found that the acoustic power radiated while exciting the soundboard at single points is strongly affected by vibration modes up to $500 \mathrm{~Hz}$.

Kindel and Wang [7] described one of the first Finite Element (FE) models of piano soundboard: by using a small number of shell and beam elements the first few modes of the soundboard were obtained. The piano structure was modelled via beam elements and the vibration modes up to $130 \mathrm{~Hz}$ were compared with experiments. Kindel and Wang also discussed the importance of modelling the rim of the piano to obtain the correct series of modes at low frequencies. Conklin [8] presented various examples of modal testing and mechanical impedance measurements. Different aspects of soundboard design were touched in that review paper; of particular interest are the description of the role of the bridge, which reduces the point mobility at the strings connection points (if compared to the bridgeless board), the effects of string tension and the presence of ribs making the soundboard behave as a periodic structure with periodic attenuations.

In the 1990s Giordano et al. developed a complete model of the piano [4, 9-12]: the main idea was that of using the Newton's law to describe the energy flow in the piano in the time domain. The soundboard's behaviour was modelled by solving, via finite differences, the equation of motion of a thin orthotropic plate. More recently another complete computational model of the piano was presented by Chabassier et al. [13]. In this case the soundboard was modelled as a Reissner-Mindlin plate with space variable mechanical properties, so that variable thickness and variable ribs spacing could be accounted for.

Berthaut et al. [14] described an experimental modal analysis campaign performed on a grand piano soundboard in free-edge boundary condition along with a corresponding Fe model. $\mathrm{Nu}$ merical results were compared with experiments and a good correspondence was found up to nearly $250 \mathrm{~Hz}$. Moore and Zietlow [15] developed a method of electronic speckle pattern interferometry in order to measure the soundboard deflection shapes. The comparison presented between their measurements and a simplified FE model of the structure showed that the vertical force exerted by the strings on the soundboard has a non-negligible effect on the natural frequencies of the lowest modes, while it can be neglected when working in the mid/high frequency range. Mamou-Mani et al. [16] presented a FE model of the soundboard to study the effect of the crown and downbearing. It was observed that nonlinear solution is required when the effect of these two parameters have to be taken into account. Results were presented in the frequency range corresponding to individual mode resonances, i. e. up to $450 \mathrm{~Hz}$. These outcomes were later experimentally confirmed [17]. In a recent study Chaigne et al. [18] focused on the influence of the ribs spacing and showed that above $1 \mathrm{kHz}$ there is a localisation effect which is very sensitive to small changes in the spacing itself.

Two papers ([19, 20]) were published by Ege and Boutillon on the vibroacoustics of piano soundboards in 2013. In [19] the soundboard was excited with a loudspeaker and the non-linear response of the soundboard was estimated to be $30 \mathrm{~dB}$ to $50 \mathrm{~dB}$ below the linear one. The measurement was performed for vibration levels of the soundboard corresponding to a musical $f f$ (fortissimo). This result confirms that linear vibration models are sufficient to represent the most important vibroacoustic features of piano soundboards. In the same paper the authors applied a novel modal analysis technique [21] and estimated natural frequencies and damping ratios up to $3 \mathrm{kHz}$ and mode shapes up to $500 \mathrm{~Hz}$. By studying modal density the authors found that below $1.1 \mathrm{kHz}$ the soundboard behaves similarly to a homogeneous plate while above $1.1 \mathrm{kHz}$ the modal density tends to be that of the strips defined by the ribs. A finite element model confirmed that in this frequency range modes are localised between ribs. In Ref. [20] the same authors introduced a simplified model of the soundboard. The main structure was modelled as four sub-plates at frequencies below $1 \mathrm{kHz}$ while it was divided in orthotropic strips at higher frequencies. Formulae derived from these models were used to calculate modal densities, point mobilities and dispersion curves. Good agreement was found between the average point mobilities calculated with this approach and measurements from literature. 

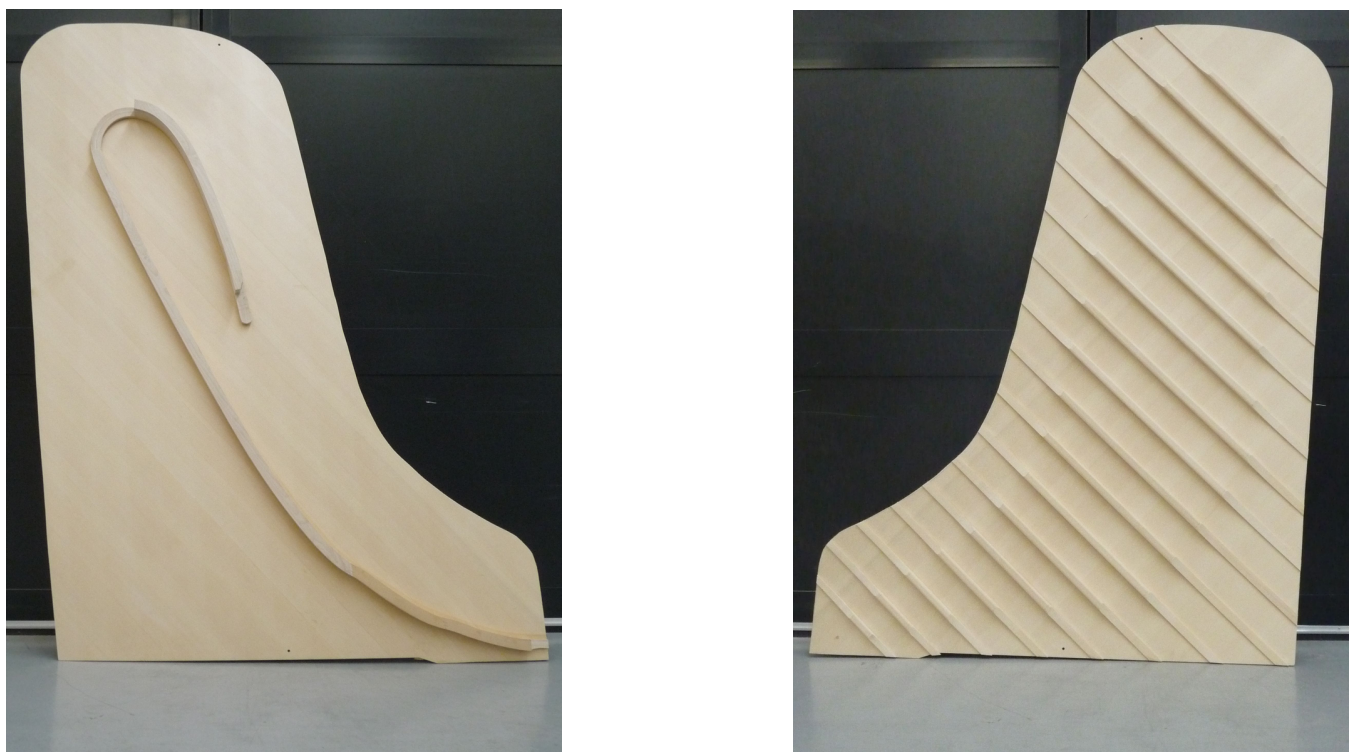

Figure 1: Upper and lower view of a Fazioli F278 soundboard at manufacturing STAGE 2

This paper illustrates the main results of a research project aimed at developing a vibroacoustic numerical model of the soundboard, to be used as a support for instrument design. This paper builds upon some of the results already published in [22] and [23], but it includes significant extensions and advances: an additional measurement and modelling stage, a more detailed description of the procedure adopted to setup the FE model of the soundboard, and new results relevant to point mobility at the bridge.

Attention is focused on the experimental modal analysis carried out on the soundboard of a Fazioli F278 grand piano (Fig. 1) and on the FE model that has been developed in parallel. The main idea behind the authors' research is that accurate FE modelling is important for developing a predictive vibroacoustic simulation tool. Moreover a detailed structural model, that includes also the manufacturing process, is valuable if it has to be used as an effective design aid. A step-by-step procedure has been adopted to validate the FE model. Modal analysis tests are performed at four significant stages of the soundboard manufacturing process: free soundboard before bridge gluing, free soundboard after bridge gluing, soundboard glued on the rim (before stringing and tuning), and the finished instrument, complete with iron frame and tuned strings. For reasons of space, in the present paper results for the first three steps only will be presented.

The step-by-step approach has been adopted for two main reasons. The first is that it allows investigating on how the single phases of the piano manufacturing process influence the vibration properties of the soundboard. The second and most important reason is related to the objective of simplifying the development of the soundboard FE model: by performing a stepby-step validation, the model is expected to reach better reliability and accuracy. To this end, the development of the FE model proceeds in parallel to the experimental tests, by reproducing the soundboard configuration at the same four manufacturing stages. The FE model takes into account the tapering as well as the curvature of the board. Peculiar to the presented approach is that the soundboard curvature is not assumed a priori but it is obtained by explicitly simulating the manufacturing process. As a side effect, the presence of the induced self-stresses is also considered.

Some details on the manufacturing process are given in Sect. 2, while experimental testing is described in Sect. 3. The FE model and the parameter updating algorithm are illustrated in Sect. 4 and 5. Finally, experimental and numerical results are compared in Sect.6

\section{Manufacturing stages}

Four main phases of the soundboard manufacturing process are singled out to be simulated by $\mathrm{FE}$ analysis and experimentally investigated. At the end of each manufacturing phase the soundboard is at a "production stage" numbered from STAGE 1 to STAGE 4 . The very first production step defines a stage 0 , corresponding to the starting configuration of the analysis.

\subsection{Preliminary preparation, STAGE 0 .}

The construction of the F278 soundboard starts by edge gluing quarter-sawn small planks of Norway spruce (picea abies); the resulting panel is then shaped, planed, seasoned, and finally tooled by a Computer Numerically Controlled (CNC) milling machine to achieve a carefully controlled variable thickness. This process is called "diaphragming" in the piano industry, a term which refers to the thinning of the soundboard near the edges. The use of quarter-sawn planks guarantees that the wood anatomical directions are almost constant across the panel: longitudinal and radial $(\mathrm{L}, \mathrm{R})$ in the plane of the soundboard, tangential (T) normal to the surface. In Fig. 1 the $\mathrm{L}$ direction can be recognised from the wood grain. 


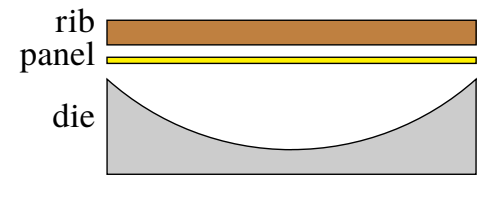

a)

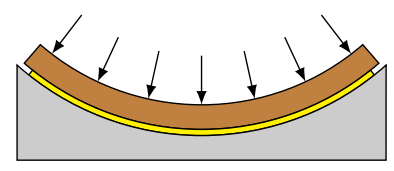

b)

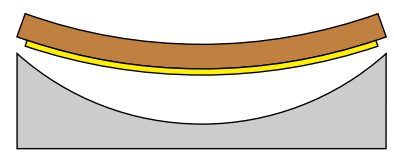

Figure 2: Compression crowning by gluing straight ribs to a flat panel in a curved die. (a) pressure is applied on the ribs while liquid adhesive allows rib-panel slip; (b) after adhesive curing, pressure is removed and a residual curvature is obtained due to rib-panel stick. The curvature of the die is exaggerated for clarity.

\subsection{Gluing of the ribs and crowning, STAGE 1.}

In the finished piano the soundboard is subjected to a considerable load due to the tension and the angle of the strings across the bridges. This load is normal to the soundboard surface and its value is typically 0.5 to $3 \%$ of each string tension [8]; for the piano under study it has a resultant of about $3 \mathrm{kN}$. To withstand this load and to avoid an excessive deformation, the soundboard has to be stiffened by a system of ribs on the surface opposite to the bridges. Spruce has a very low stiffness in the cross grain direction (see Tab. 2): therefore the ribs have to run perpendicular to the spruce grain. The ribs themselves are made of spruce, with grain parallel to their axis.

In order to better control the deflection of the soundboard under the load of the tensioned strings, the ribs are assembled to the panel by means of a peculiar procedure that causes an initial reverse deflection, called "crown" in the piano industry. There are several methods for obtaining the desired crown, the most simple one consisting in using curved ribs, i. e. "rib crowning". For the soundboard under investigation a different procedure is adopted, based on initially straight ribs. The ribs are glued to the panel in a special press with a curved die. When pressure is applied, the adhesive is still liquid and both ribs and panel are free to assume the shape of the die; as it is clear from Fig. 2 this is accompanied by a relative sliding between panel and ribs. After adhesive curing any further slip is prevented; therefore, when pressure is removed elastic springback occurs but ribs and panel cannot return to the initial straight and flat configuration. As a consequence, the soundboard shows a residual curvature and a residual self-stress field. Owing to the low bending stiffness of the panel in the cross grain direction, its internal stresses are mainly compressive, while bending dominates in the ribs. For this reason the method is called compression crowning. (Other crowning methods introduce mainly bending in the panel, see [24].)

The initial geometry of panel, ribs and die is known but the final "crowned" shape and the self-stress field are unknown. Both these two features can affect the vibroacoustic properties of the soundboard, and hence the tonal quality of the finished piano. While the final shape can be measured, internal stresses are hard to assess experimentally. In this paper a method is proposed to estimate these by a FE simulation of the gluing process.

\subsection{Gluing of the bridges and planing down of the ribs, STAGE 2.}

The bridges are glued to the opposite side of the panel, with a process that closely resembles the ribs assembly, but the curvature of the die is chosen to be not so different from the curvature of the soundboard at STAGE 1 . Therefore one has to expect only a slight modification of both the curvature and self stress state. Finally the ribs are planed down at the ends, to obtain their final shape: during this process self-stresses are partially released, but the curvature of the soundboard increases, owing to the reduced stiffness of the ribs. Fig. 1 shows the soundboard at STAGE 2.

Again, while the soundboard shape at STAGE 2 can be measured, the self-stress state is unknown: therefore both bridge gluing and ribs planing have been included into the FE simulation.

\subsection{Final assembly, stage 3 and stage 4.}

From the manufacturing point of view, at STAGE 2 the construction of the soundboard itself is concluded and the remaining steps involve assembly into the complete piano. However during assembly new constraints are imposed and loads are applied to the soundboard, that may cause the vibrational properties to change. For the purpose of the present investigation two further stages are defined.

At STAGE 3 the soundboard is assembled in the piano case by gluing its edge on the internal rim. The internal rim itself is fitted to the curved shape of the soundboard, in order to prevent additional stresses due to the new constraints. The case is much stiffer than the soundboard and in first approximation can be assumed rigid ${ }^{2}$ STAGE 4 corresponds to the finished piano. The strings exert a downward force which causes a deflection of the soundboard called "downbearing" and the crown is consequently reduced. This phenomenon changes the vibration properties of the soundboard (see [16]).

\section{Experimental results}

\subsection{Test procedure}

The experimental tests presented in this paper were carried out on the same piano soundboard at manufacturing stages 1 to 3. In particular, at the Fazioli factory in Sacile (Italy), the soundboard at staGE 1 (before bridge gluing), see Fig. 3(a) and STAGE 2 (after bridge gluing) was tested by suspending it on a steel cable so as to reproduce a free-edge boundary condition.

\footnotetext{
${ }^{2} \mathrm{~A}$ more detailed analysis shows that its flexibility is expected to have some influence on the soundboard natural frequencies, especially for low order modes [7].
} 
The length of the cable was chosen so as to set the system first natural frequency below $2 \mathrm{~Hz}$. This choice allowed the soundboard rigid motion and its flexible vibration modes to be fully decoupled. The soundboard was instrumented with 10 piezoelectric uniaxial accelerometers (sensitivity $10 \mathrm{mV} \mathrm{s}^{2} \mathrm{~m}^{-1}$, range $10000 \mathrm{~m} \mathrm{~s}^{-2}$, bandwidth $10 \mathrm{kHz}$, mass $2 \mathrm{~g}$ ). The tests were performed using two different impact hammers: first a large impact hammer (160 g mass) with a soft tip allowed investigating the low/mid frequency range (up to $1 \mathrm{kHz}$ ), then the impact tests were repeated using a small hammer ( $5 \mathrm{~g}$ mass) with an harder tip, to excite higher frequencies. By means of the latter device it was possible to introduce enough energy into the system so as to measure the soundboard Frequency Response Function (FRF) up to approximately $5 \mathrm{kHz}$. The tests were repeated for two excitation points and six different accelerometer positioning, so as to cover a regular measurement grid with 60 nodes in total, equally spaced on a $200 \mathrm{~mm} \times 200 \mathrm{~mm}$ square mesh ${ }^{3}$

Analogous tests were performed for stAGE 3 (see Fig. 3(b)], at the Department of Mechanical Engineering of Politecnico di Milano. Due to the higher weight of the structure under test, the piano case was resting on three supports located under the rim, so as to weakly affect the soundboard vibration behaviour. During STAGE 3 , in addition to the accelerometers positioned on the $200 \mathrm{~mm} \times 200 \mathrm{~mm}$ measurement grid, an interferometric laser velocimeter (Polytec PSV-400, with adjustable sensitivity and measurement range) was used to scan a $100 \mathrm{~mm} \times 100 \mathrm{~mm}$ grid.

The $H_{1}$ estimator ([25]) was adopted to derive the soundboard FRF's from the collected experimental data:

$$
H_{j k}(\omega)=\frac{G_{j k}(\omega)}{G_{k k}(\omega)},
$$

where $G_{k k}$ is the autospectral density function of the input force $q_{k}, G_{j k}$ the cross-spectral density function between the input force $q_{k}$ and the output vibration $x_{j}$, and $H_{j k}$ the $H_{1}$ estimate of the FRF between $q_{k}$ and $x_{j}$. The coherence function $\gamma_{j k}^{2}$ between $q_{k}$ and $x_{j}$ was calculated too, which provides a measure for the quality of the FRF data:

$$
\gamma_{j k}^{2}(\omega)=\frac{\left|G_{j k}(\omega)\right|^{2}}{G_{j j}(\omega) G_{k k}(\omega)} .
$$

The calculation of $G_{j j}, G_{k k}$, and $G_{j k}$ was carried out according to the procedure indicated by Bendat and Piersol [26], which consists in computing the Fast Fourier Transform (FFT) for each block of sampled data (corresponding to one single impact test), then the corresponding raw autospectral/cross-spectral density functions for each $j-k$ node pair, and in finally averaging over the data blocks corresponding to the ten impact test repetitions, for fixed $j$ and $k$.

Attention is first focused on a frequency range limited to $1 \mathrm{kHz}$ to show detailed results on natural modes identification (Section 3.2, while examples of bridge point mobilities up to

${ }^{3}$ Under the same assumptions made below for estimating the soundboard mobility at low frequency, see Sect. 3.3 Eq. 3, the structural wavelength at $400 \mathrm{~Hz}$ is about $590 \mathrm{~mm}$.
$3 \mathrm{kHz}-5 \mathrm{kHz}$ are presented in Sect. 3.3 , for the different manufacturing stages (STAGE 1 to STAGE 3 ).

Two examples of mobility FRF's and associated coherence functions are reported in Figs. 4(a) and 4(b), which refer to the measurements carried out at STAGE 1 and STAGE 3 , for the same pair of excitation and measurement points (both located close to the bridge, in correspondence with the positions where the bridge itself is crossed by the $\mathrm{A} \sharp_{4}$ and $\mathrm{D} \sharp_{3}$ strings respectively). In this case, the soundboard was excited by the small hammer and the output vibration was measured by means of a piezoaccelerometer. However, during the tests, it was checked that the FRF's obtained by different combinations of the excitation and measurement equipment were in very good agreement, so as to be confident with the reliability and accuracy of the measurement process.

\subsection{Modal analysis}

The identification procedure used for the estimation of modal parameters from experimental FRF's consists in a two-step approach.

First, the Least Squares Complex Exponential (LSCE) algorithm [27,-29] is applied to estimate the system poles and the corresponding natural frequencies $\omega_{n}$ and damping ratios $\xi_{n}$. This is a time-domain algorithm which requires as input the $I m$ pulse Response Functions (IRF's) for all the considered $j-k$ pairs. The IRF's are obtained by computing the inverse FFT of the FRF data. Typically the LSCE estimator is applied assuming different model order: therefore a stabilisation chart is obtained [27, 29], which allows selecting the system physical poles considering their stability for increasing model order.

The second step consists in the classical FRF-based curvefitting method [25]: according to the idea of modal superposition, the measured FRF's are fitted with an analytical model consisting of a series of second order systems. Least squares minimisation operates in a user-defined frequency band which includes a certain number of vibration modes: a limited variation of their natural frequencies and damping ratios with respect to the previous LSCE estimates is allowed. To reach suitable curve-fit, high and low frequency residuals are added to the resonant modes [25], so as to account for the contribution of the modes not included in the selected frequency band. The adopted frequency-domain algorithm allows simultaneous processing of the whole FRF experimental data set. The final output are the modal parameters (i. e. natural frequencies, damping ratios and mode shapes) of the vibration modes included in the frequency band of interest. The reliability of the overall identification process can be finally verified by visually checking the accuracy of the FRF fitting: looking at Figs. 4(a) and 4(b), it can be concluded that the identified FRF's reproduce the experimental ones in a fully satisfactory way.

By following this procedure the following sets of vibration modes were identified: 52 modes up to $450 \mathrm{~Hz}$ for the soundboard at STAGE 1,39 modes up to $386 \mathrm{~Hz}$ for soundboard at STAGE 2,34 modes up to $348 \mathrm{~Hz}$ for soundboard at staGe 3 . The identified damping ratios $\xi_{n}$ vary from $0.4 \%$ to $1.0 \%$ for the first two stages, from $0.8 \%$ to $2.8 \%$ for the third stage. Natural 


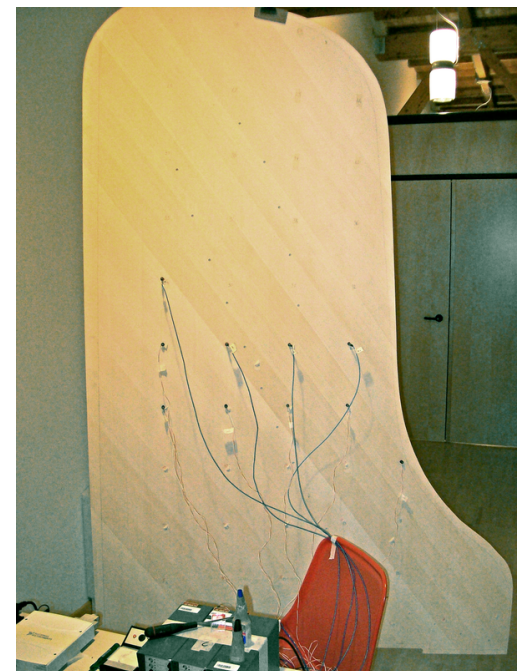

(a) STAGE 1: free soundboard before bridge gluing.

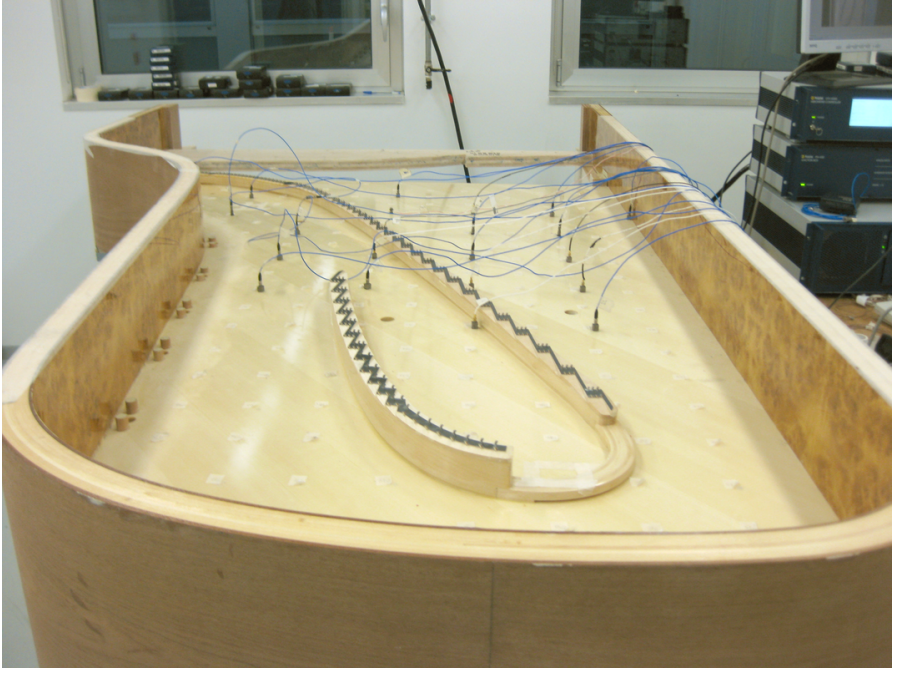

(b) STAGE 3: soundboard assembled on the piano frame.

Figure 3: Experimental modal analysis at different manufacturing stages.

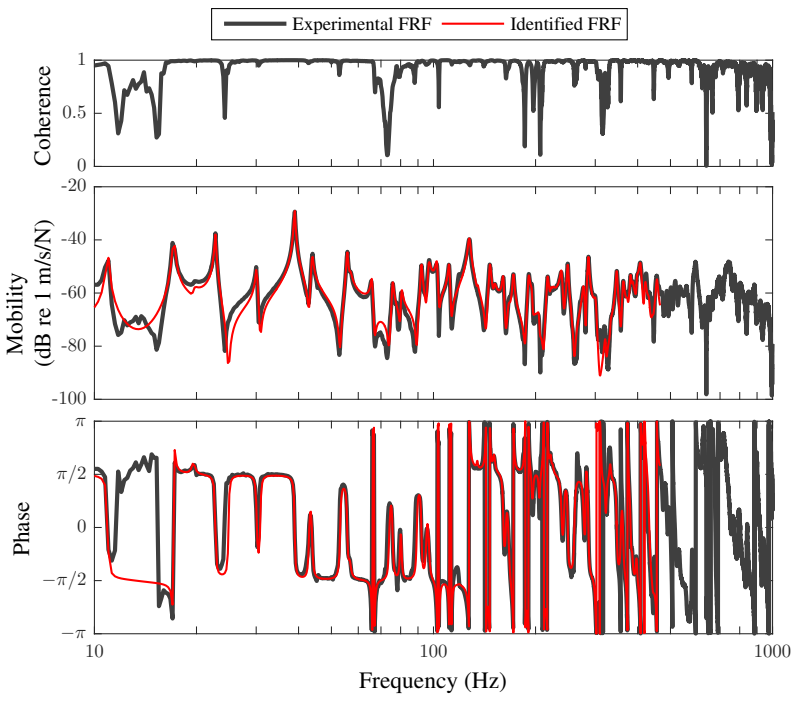

(a) STAGE 1: free soundboard before bridge gluing.

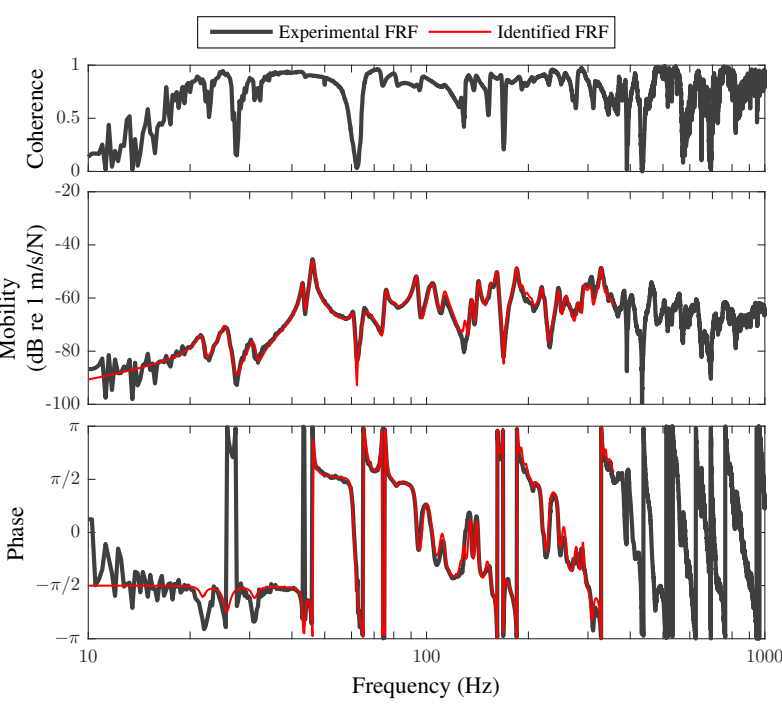

(b) STAGE 3: soundboard assembled on the piano frame.

Figure 4: Comparison between experimental and identified FRF. 
frequencies and damping ratios of the first 20 identified vibration modes are reported in Tab. 1, for stages 1 to 3 .

The three construction stages correspond to three different structures, in terms of configuration and especially boundary conditions and damping. This results in three different values of the transition frequency between the low-frequency range, which is characterised by individual modal resonances, and the mid/high-frequency range, which shows coupled multi-mode response. Identification algorithms for modal parameters estimation can be efficiently applied only in the low-frequency range, were the overlap factor [21] is limited, i. e. modes are clearly spaced and the system shows limited damping. In Figs. 4(a) and 4(b) the identified FRF's, for STAGE 1 and STAGE 3 are plotted only up to the transition frequency mentioned above.

\subsection{Point mobilities at the bridge}

To show how the coupling between the strings and the soundboard varies during the manufacturing process, it is useful to analyse the bridge point mobilities at STAGE 1, STAGE 2 and STAGE 3 . The selected measurement point is located at the position where the strings of note G3 are connected to the bridge. At STAGE 1, when the bridge is not yet glued, this corresponds to a position between two ribs.

Fig. 5(a) shows the magnitude diagram of the point mobility at STAGE 1 . The mean value is constant up to $700 \mathrm{~Hz}$ and increases with a slope of about $15 \mathrm{~dB}$ per octave thereafter. Around $1.5 \mathrm{kHz}$ the curve has a plateau and then drops slightly. As a mean of comparison two lines representing the mobilities of infinite structures are also added to the figure. This is done by following the mean value theory proposed by Skudrzyk [30] and recently applied in the field of musical acoustics by many authors [31-36]. The first one represents the mobility of an infinite homogeneous plate, equivalent to the board-ribs structure. This line is obtained assuming that the overall bending stiffness of the ribs along their length is equivalent to that of the board in the grain direction. This would make the board-ribs structure an homogeneous isotropic plate with the Young modulus of spruce and a mass per unit area obtained including the mass of the board and of the ribs. Under these assumptions the point mobility of the equivalent plate is calculated as:

$$
Y_{\mathrm{pl}}=\left(8 h_{\mathrm{eq}}^{2} \sqrt{\frac{E \rho}{12\left(1-v^{2}\right)}}\right)^{-1}
$$

where $h_{\mathrm{eq}}$ is the equivalent thickness that corresponds to the overall mass, and is equal to $12.8 \mathrm{~mm}$ in this case, $E$ is the Young modulus, set to $14 \mathrm{GPa}, \rho$ is the wood density $\left(450 \mathrm{~kg} / \mathrm{m}^{3}\right)$ and $v$ the Poisson ratio (equal to 0.36 ). The average value of the measured point mobility is well represented by this equivalent homogeneous plate up to $300 \mathrm{~Hz}$.

Additionally, the mobility of an infinite plate having the structural properties of the board alone (without ribs) is also shown (dashed line in Fig. 5 (a)). The calculation is as in Eq. 3 setting the thickness to $8 \mathrm{~mm}$. In this case the orthotropy is strong and an equivalent Young modulus is obtained from the geometric mean of the two directions (14.6 GPa and 0.69 GPa). The so computed mobility can be considered a rough estimate of the actual soundboard mobility, under the assumption that at high frequency (above $\approx 1 \mathrm{kHz}$ ) the soundboard itself behaves like the strip contained between two adjacent ribs. A more detailed methodology for wide-band mobility analysis can be found in Boutillon [36].

Fig. 5. (b) shows the point mobility on the bridge for staGE 2 and STAGE 3 . The stiffening effect due to the changes in boundary conditions (from free to clamped edge) is clear only below $200 \mathrm{~Hz}$. The first soundboard natural frequency is shifted from $12 \mathrm{~Hz}$ to $46 \mathrm{~Hz}$ and only few modes exist in STAGE 3 below $200 \mathrm{~Hz}$. Above $200 \mathrm{~Hz}$ the two lines show similar trends and the mean value of both decreases at roughly $5 \mathrm{~dB}$ per decade up to $2 \mathrm{kHz}$ to $3 \mathrm{kHz}$. Above these values both mobilities increase slightly and maintain a constant value of roughly $-60 \mathrm{~dB}$ up to $5 \mathrm{kHz}$. Above $5 \mathrm{kHz}$ the mobility measured at STAGE 3 drops again at the steeper rate of nearly $20 \mathrm{~dB}$ per octave. The equivalent result for STAGE 3 is not shown here, due to the poor coherence above $5 \mathrm{kHz}$. In this case also, the mobility of an equivalent infinite structure is calculated for comparison. This is done by adding the impedances (reciprocal of mobility) of the infinite beam equivalent to the bridge and of the equivalent plate presented above [30]:

$$
Y_{\mathrm{sb}}=\left(8 h_{\mathrm{eq}}^{2} \sqrt{\frac{E \rho}{12\left(1-v^{2}\right)}}+2(1+i) \sqrt{\omega}(E I)^{\frac{1}{4}}(\rho A)^{\frac{3}{4}}\right)^{-1}
$$

where $\omega$ is the circular frequency and $I$ is the moment of inertia of a rectangular section of area $A$. $A$ and $I$ are obtained from the bridge geometry at this position, which is $32 \mathrm{~mm}$ wide and $37 \mathrm{~mm}$ thick. The other parameters are the same as in Eq. 3 The mean value of the measured mobility at STAGE 2 and STAGE 3 is in good agreement with the one predicted with this simplified approach up to $2 \mathrm{kHz}$ to $3 \mathrm{kHz}$.

\section{Finite Element model}

As outlined in Sect. 2, the construction of a soundboard is quite complex: the geometry and the self-stress state attained at each stage are the outcome of the preceding manufacturing procedure. Since geometry and self-stress state are not known a priori, at each manufacturing stage a "standalone" FE model cannot be used to predict the actual natural frequencies, but has to be tuned against experimental data [14]. To overcome this difficulty in the present paper a different FE modelling strategy is pursued: instead of creating a sequence of "stage" models, each with its own tuning problems, a direct simulation of the whole manufacturing process is adopted. By doing so the "model state" and its modal properties at each stage can be included in the model.

Model tuning is performed just once at STAGE 1 against measured natural frequencies and modes, thus updating a subset of the material elastic constants of Tab. 2. At subsequent stages (STAGE 2 and following) no further tuning is performed. Natural frequencies extraction, tuning, and material properties updating are described in Section 5 . 
Table 1: Frequencies and damping ratios of the first 20 identified vibration modes at STAGE 1 to STAGE 3.

\begin{tabular}{cc|cc|cc}
\hline \multicolumn{2}{c|}{ STAGE 1 } & \multicolumn{2}{c|}{ sTAGE 2 } & \multicolumn{2}{c}{ STAGE 3 } \\
\hline nat. freq. & damping & nat. freq. & damping & nat. freq. & damping \\
$(\mathrm{Hz})$ & ratio (\%) & $(\mathrm{Hz})$ & ratio $(\%)$ & (Hz) & ratio (\%) \\
\hline 7.5 & 0.6 & 11.9 & 0.7 & 43.1 & 0.8 \\
10.9 & 0.6 & 19.0 & 0.6 & 46.0 & 1.0 \\
17.1 & 0.6 & 28.2 & 0.5 & 60.8 & 1.1 \\
22.7 & 0.4 & 32.9 & 0.6 & 65.7 & 2.8 \\
30.1 & 0.5 & 47.9 & 0.5 & 75.7 & 1.0 \\
38.9 & 0.4 & 50.5 & 0.7 & 81.3 & 1.7 \\
43.8 & 0.7 & 61.1 & 0.9 & 93.5 & 1.2 \\
55.7 & 0.5 & 63.3 & 0.6 & 103.9 & 2.2 \\
56.7 & 1.0 & 70.9 & 0.6 & 112.9 & 1.8 \\
65.9 & 0.6 & 77.7 & 0.5 & 126.3 & 1.2 \\
74.3 & 0.6 & 85.6 & 0.6 & 135.9 & 0.9 \\
75.8 & 0.5 & 89.9 & 0.8 & 142.3 & 0.9 \\
80.5 & 0.5 & 100.7 & 0.5 & 151.5 & 1.4 \\
92.1 & 0.5 & 105.8 & 0.7 & 153.6 & 0.9 \\
96.9 & 0.8 & 11.4 & 1.0 & 157.1 & 1.5 \\
102.4 & 0.6 & 125.9 & 0.8 & 161.5 & 0.9 \\
111.2 & 0.5 & 131.4 & 0.6 & 170.9 & 1.4 \\
119.1 & 0.7 & 139.1 & 0.5 & 174.8 & 1.3 \\
127.5 & 0.9 & 153.3 & 0.6 & 185.0 & 1.1 \\
134.8 & 0.5 & 167.1 & 0.9 & 191.2 & 1.2 \\
\hline
\end{tabular}
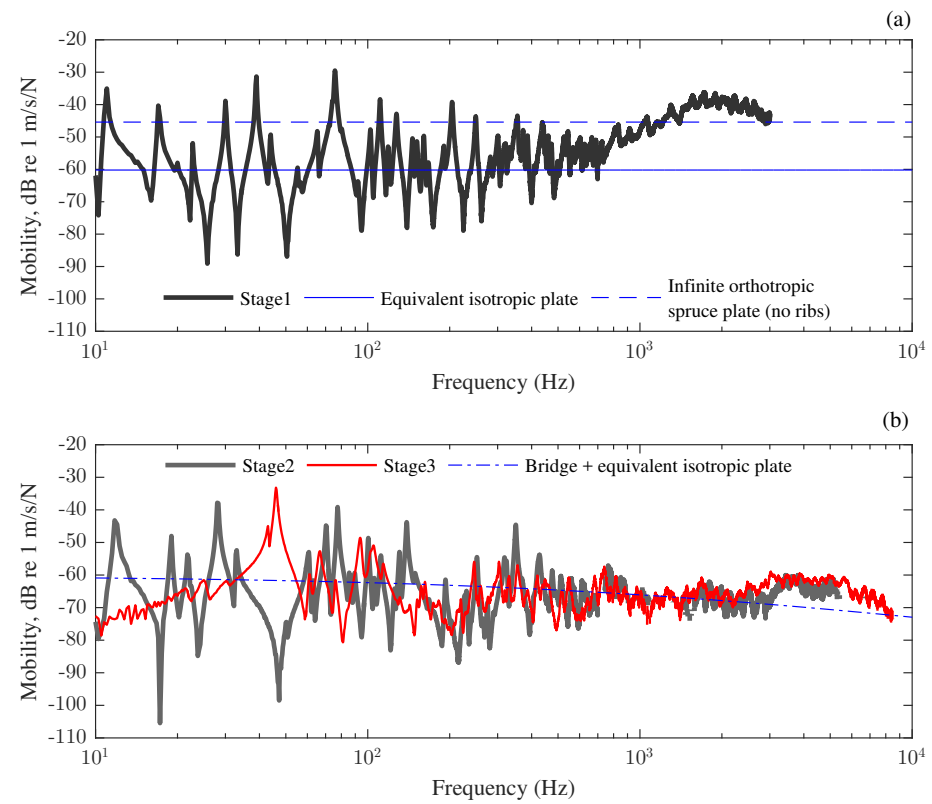

Figure 5: Measured point mobilities at Stage 1, stage 2 and stage 3. (a) Point mobility at the position of note G3 at stage 1 (without bridges). The measurement point is located between two ribs. The mobility of an infinite isotropic plate (continuous line), equivalent to the board-ribs structure, and that of an infinite orthotropic plate (dashed line), equivalent to the board alone, are shown for comparison. (b) Point mobility at the position of note G3 at STAGE 2 and sTAGE 3. The measurement point is located on the bridge. The mobility of the bridge (infinite beam) combined with the infinite equivalent plate is shown for comparison (dash-dot line). 
The manufacturing process has been modelled through of a fully nonlinear FE model, i. e. taking into account finite strain, arbitrary displacement and stress-stiffening effects. During the whole manufacturing process dynamic effects are negligible, so that a quasi-static calculation is appropriate. Material behaviour can be considered linear (see Eq. (A.1) and Tab. 2) due to the small loads involved. Creep effects have been neglected.

All FE analyses were carried out using the Abaqus/Standard software v. 6.10. This is a general-purpose nonlinear FE code that is organised around the concept of an "analysis problem history" defined in terms of "steps". Each step is associated with an "analysis procedure" that defines the type of analysis to be performed, [37]. This approach maps nicely to the present problem in which the "manufacturing history" of the soundboard has to be simulated. Since the problem is quasi-static, the Abaqus "static stress analysis" (keyword $*$ STATIC) procedure has been used throughout the analysis. In order to account for the geometric nonlinearities linked to a large-displacement formulation, the *STEP, NLGEOM=YES keyword was specified.

In order to define analysis steps corresponding to the manufacturing process, a careful analysis of both the physical process and the FE numerical convergence issues has to be performed. (In a nonlinear FE simulation "large steps" in which many different processes and transformation occur tend to have grater convergence problems than "small steps" in which the state change is limited.) For the study presented in the present paper, a rather fine grained approach was adopted: the manufacturing is split into many smaller steps, in order to have a better control on the numerical convergence, at the cost of a more complex model setup. Within this approach, a manufacturing STAGE is simply the state of the FE model at the end of a given key analysis step.

\subsection{FE model at STAGE 0}

The soundboard at STAGE 0 is the starting point of the present analysis, i. e. the reference (initial) state of the FE model.

At STAGE 0 the soundboard is modelled as a stress-free, variable thickness, flat solid plate made of an homogeneous material. In reality the soundboard is obtained by gluing together smaller wooden planks, but the inhomogeneities intrinsic to wood and those due to the not perfect alignment of the anatomical directions between different planks are not relevant at the macroscopic scale. Moreover the adhesive has a negligible thickness with respect to the planks in-plane dimensions but still it guarantees a very strong bonding, so that no stress/strain discontinuities have to be expected along the adhesion planes. Finally no internal stresses are present after the first fabrication steps that lead to STAGE 0 .

Wood is a complex natural material, but to the purposes of the present analysis it can be modelled as an homogeneous orthotropic elastic material, with the anatomical directions (L, R, T) aligned to the elasticity principal axes $(1,2,3)$ [38]. According to equation A.1, an orthotropic material is characterised by the nine elastic parameters $E_{1}, E_{2}, E_{3}, v_{12}, v_{13}, v_{23}, G_{12}, G_{13}$, and $G_{23}$; moreover the mass density $\rho$ has to be considered for modal analysis. It is easy to understand that determining these nine moduli plus mass density is a hard task, since they can be defined only in a statistical sense: wood from different logs has different properties, owing to the varying micro and macro climatic conditions that affect tree growth. Even specimens from the same log, but taken from different annual rings exhibit different material properties. Finally the same specimen changes its properties according to moisture content, seasoning, rate of loading.

Instead of trying a direct measurement of these ten material properties, an initial educated guess was made about their value, based on the data available from the literature (mainly from [38, [39]); the chosen values are listed in Tab. 2, under the column "initial". As it will be made clear in the sequel (Sect. 5) these initial values were updated by comparing the predicted natural frequencies to the measured ones, so that their precise initial estimate is not critical.

For an appropriate choice of the soundboard finite element types, one has to consider that the gluing process between the lower/upper face of the soundboard with the ribs/bridges has to be simulated. Moreover, due to diaphragming, the thickness is not constant across the board. For these reasons a solid model is convenient, but still, due to the small thickness, a thin shell model is adequate. This is why Abaqus "Continuum Shell" elements SC8R [37] were chosen for modelling the soundboard. About 6000 elements where used, with an average in-plane edge $\approx 20 \mathrm{~mm}$. The element size was chosen in order to have at least five elements along the wavelength at $4 \mathrm{kHz}$ of an infinite isotropic plate $8 \mathrm{~mm}$ thick, with a Young modulus $E=\sqrt{E_{1} E_{2}}$, where $E_{1}$ and $E_{2}$ are the orthotropic Young moduli of Norway spruce, Tab. 2. Indeed at high frequency vibration modes are localised between two ribs [18, 19], and the mesh size has to be fine enough to represent these mode shapes.

The board thickness was obtained directly from the same CAD models used for generating the working profiles of the diaphragming CNC milling machine.

\subsection{FE model at STAGE 1 and STAGE 2}

STAGE 1 and STAGE 2 essentially involve two types of procedures:

- ribs and bridge gluing and

- ribs planing down.

\subsubsection{Ribs and bridge gluing}

The main idea is to handle the gluing process by simulating a contact problem without an explicit model of the adhesive layer. When the adhesive is still liquid one has frictionless unilateral "hard" contact between the surfaces to be glued; this means that no penetration (overclosure) is allowed, relative sliding (slip) of arbitrary entity is permitted as well as surface separation (contact opening). After adhesive curing the contact conditions are changed to "tie", i. e. no separation and no sliding is permitted. The rationale for this model is that the adhesive layer is very thin and therefore the contribution to stiffness and mass of the adhesive itself can be neglected. On the other hand the bond is very strong, and in normal working conditions one does not expect any adhesive failure. 
Table 2: Wood mechanical properties. Anatomical directions (L, R, T) are aligned to orthotropy principal directions (1, 2, 3).

\begin{tabular}{|c|c|c|c|c|c|c|}
\hline & & \multicolumn{2}{|c|}{$\begin{array}{c}\text { panel } \\
\text { picea abies }\end{array}$} & \multicolumn{2}{|c|}{$\begin{array}{c}\text { ribs } \\
\text { picea sitchensis }\end{array}$} & \multirow{2}{*}{$\begin{array}{c}\text { bridges } \\
\text { acer } \\
\text { estimated }\end{array}$} \\
\hline & & initial & updtd. & initial & updtd. & \\
\hline$E_{1}$ & $(\mathrm{GPa})$ & 12.8 & 14.6 & 13.4 & 12.7 & 12.6 \\
\hline$E_{2}$ & (GPa) & 0.625 & 0.686 & 1.04 & 1.04 & 1.66 \\
\hline$E_{3}$ & (GPa) & 0.397 & 0.436 & 0.397 & 0.478 & 0.819 \\
\hline$v_{12}$ & & \multicolumn{2}{|c|}{0.36} & \multicolumn{2}{|c|}{0.37} & 0.42 \\
\hline$v_{13}$ & & \multicolumn{2}{|c|}{0.45} & \multicolumn{2}{|c|}{0.47} & 0.48 \\
\hline$v_{23}$ & & \multicolumn{2}{|c|}{0.48} & \multicolumn{2}{|c|}{0.43} & 0.77 \\
\hline$G_{12}$ & (GPa) & 0.617 & 0.677 & 0.864 & 0.998 & 1.40 \\
\hline$G_{13}$ & (GPa) & 0.587 & 0.644 & 0.830 & 0.959 & 0.793 \\
\hline$G_{23}$ & $(\mathrm{GPa})$ & 0.053 & 0.058 & 0.045 & 0.042 & 0.500 \\
\hline$\rho$ & $\left(\mathrm{kg} \mathrm{m}^{-3}\right)$ & 437. & 452. & 505. & 489. & 630. \\
\hline
\end{tabular}

Ribs gluing is performed in a curved die, see Fig. 2, which has been modelled as a rigid surface; the ribs themselves have been modelled with hexahedral, reduced integration, linear FE's (Abaqus C3D8R). On average the ribs where meshed with $3 \times 3$ elements on the cross section, with an edge of about 7 to $10 \mathrm{~mm}$. The material has been modelled as homogeneous, linear elastic, orthotropic: material properties are given in Tab. 2 as Sitka spruce.

It is worth noting that, in order to correctly represent the physical process of ribs gluing, an accurate modelling of contact conditions is essential: unilateral contact and finite sliding is present at the board/ribs and board/die interface. Moreover, in order to account for the stiffening effect of the ribs gluing induced soundboard "crown" (curvature), a finite displacement formulation has to be adopted. These assumption require the adoption of a non-linear FE procedure, see [37, 40], (Abaqus keyword $*$ STEP, NLGEOM=YES).

As it is common with FE non-linear analyses, the actual gluing of the ribs involved many different steps. In the first step, condition (a) of Fig. 2, has been simulated as a frictionless unilateral contact problem, where the ribs are subjected to a pressure load so as to keep the board/ribs system in contact with the die, Fig. 6(a). Afterwards the board-to-ribs contact condition was transformed into "tie", and the applied pressure removed: the ensuing equilibrium state is possible only with an elastic springback, but the soundboard cannot recover the original stress-free state, due to adhesion, see Fig.22(b). During the spring-back analysis, the soundboard is not constrained, and numerical instabilities may arise. In order to achieve convergence of the nonlinear FE procedure, the loading and unloading of the soundboard had to be divided into many small load increments. Pressure was applied and removed on one rib at a time, to avoid to have to solve for too severe nonlinearities in a single attempt/load increment.

The crowning at the end of the gluing process is clearly visible in Fig. 6(b) The shape obtained by the FE simulation is in remarkable good visual agreement with the shape of the actual soundboard. Also experiments described in [22] show that the predicted deflection is close to the measured one.
An analogous procedure was applied for simulating the gluing of the bridge, which is assumed for simplicity as made of maple (see Tab.2). The ensuing analysis steps are not detailed here for brevity.

\subsubsection{Ribs planing down}

The planing down procedure was simulated simply by element removal. This technique is very common in damage evolution modelling: when the material corresponding to a given finite element is "damaged" it does not contribute anymore to the structural behaviour, and it can be simply cancelled form the model. Similarly, when a portion of a rib is taken away by the planing machine, the corresponding elements are removed from the model. This reduces the stiffness of the soundboard, resulting in an increase of the crowning.

\subsection{Final stages 3 and 4}

At staGe 3 the gluing of the soundboard to the piano case is simulated. But now a simpler approach can be adopted. In fact the rim is "fitted" to the soundboard edge curved shape, so that no changes in the soundboard configuration and self-stress state are to be expected. For the sake of simplicity the piano case can be assumed to be so stiff that its presence can be simply modelled by built-in (clamped) boundary conditions along the soundboard edge. As already noted, this assumption will introduce some error on the estimate of the soundboard natural frequencies, especially in the low frequency range. Better results could be obtained by coupling the soundboard model to a FE model of the case itself, but this approach will not be pursed in this paper.

Finally for STAGE 4 , the load of the strings can be simulated as a set of concentrated loads on the bridges, one for each note. If stress-stiffening effects are accounted for, this will result in a change of the soundboard eigenfrequencies, as already noted in [16] ${ }^{4}$

\footnotetext{
${ }^{4}$ Results for STAGE 4 will not be presented in the present paper.
} 


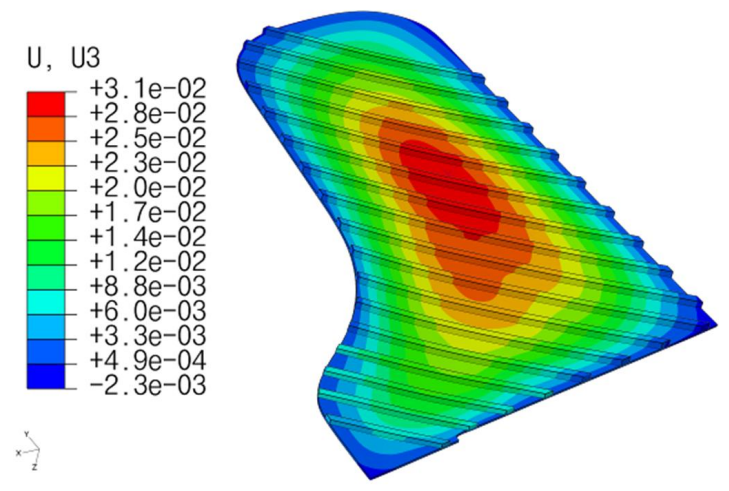

(a) No adhesion, pressure applied, board and ribs in die.
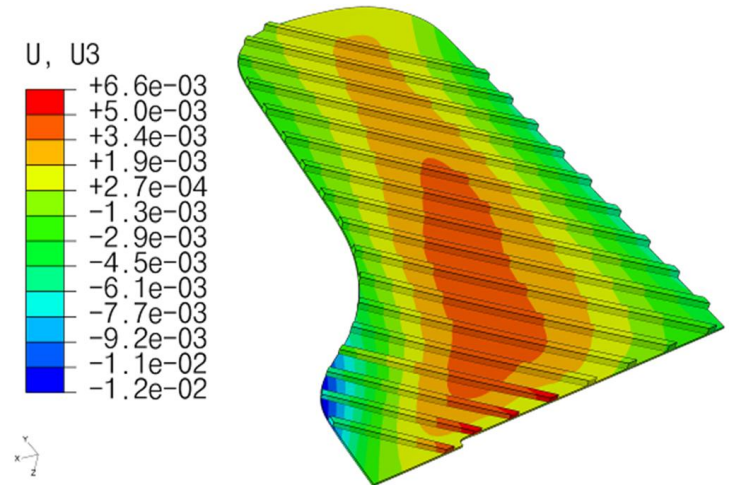

(b) After glue adhesion, pressure removed, and elastic springback out of the die.

Figure 6: Simulated ribs gluing procedure. Displacement field in the direction normal to the board, units are (m).

\section{Natural frequency extraction and material properties up- dating}

For a nonlinear FE method the equilibrium equations are not expressed in terms of a stiffness matrix but directly from the principle of virtual works, as a balance between nodal equivalent external forces and nodal equivalent internal forces. In order to define natural frequencies and modes a linearisation of the equilibrium equations is necessary, in order to define a tangent stiffness matrix, [40, 41]. More precisely, given a sequence of nonlinear steps $\left\{\mathcal{S}_{i}\right\}$, linearisation at the end of each step allows to compute the tangent stiffness matrix $\mathbf{K}_{\mathrm{T}}^{\mathcal{S}_{i}}$; when a finite displacement formulation is used, like in the present case, the mass matrix has to be computed with reference to the current reference configuration, giving raise to a $\mathbf{M}^{\mathcal{S}_{i}}$ that changes with $\mathcal{S}_{i}$. Eigenfrequencies and eigenmodes at step $\mathcal{S}_{i}$ are therefore defined from the generalised eigenproblem

$$
\left(-\omega_{\mathcal{S}_{i}}^{2} \mathbf{M}^{\mathcal{S}_{i}}+\mathbf{K}_{\mathrm{T}}^{\mathcal{S}_{i}}\right) \mathbf{u}_{\mathcal{S}_{i}}=\mathbf{0} .
$$

From the above equation it is evident that the computed eigenfrequencies and eigenmodes change with $\mathcal{S}_{i}$. Note that no damping is introduced into the FE model, so that real eigenvalues/eigenvectors are computed. However since large displacement formulation is used $(*$ STEP, NLGEOM=YES Abaqus keyword) the computed eigenfrequencies will take into account the actual curved geometry (crown and downbearing) and internal stress state.

To summarise, analogously to the experimental modal analysis results, where one has different natural frequencies at different manufacturing stages, the FE computed natural frequencies will change as the analysis history proceeds: by defining the key analysis steps, it is possible to predict the natural frequencies numerically at different manufacturing stages.

The outcome of the eigenfrequency analysis strongly depends on the material properties, the initial estimates of which are given in Tab. 2. Considering all the uncertainties in determining wood properties by means of direct measurement, the material parameters were tuned so as to best fit the computed eigenmodes on the corresponding experimental data at STAGE 1.
Indeed, starting from a tentative value of the material parameters, a set of natural frequencies and normal modes can be obtained. These numerical values are compared to the experimental ones, and the elastic material properties are updated by applying an error minimisation technique, as outlined in Sect.5.2.

It should be noted that at STAGE 1 the soundboard is a composite structure: a Norway spruce slab with Sitka spruce ribs. Therefore the parameters of two coupled materials have to be identified. This could be considered a disadvantage, since usually one tries to identify parameters of a single material at a time, e. g. by analysing STAGE 0 (i. e. tuning numerical frequencies and modes of the Norway spruce panel with no ribs against experimental modal data) and devising a specific procedure for the ribs alone. However, for the purpose of the present investigation, material parameter identification at STAGE 1 was preferred for several reasons.

- STAGE 0 is a completely different structure from the soundboard at STAGE 1: the unribbed board is strongly anisotropic and the vibrational response is expected to be completely different from the ribbed soundboard. Therefore it would have been of little interest to compare sTAGE 0 with the subsequent ones.

- The identification procedure at STAGE 1 gives a better insight on how the choice of the soundboard materials affects its vibrational properties (see the design sensitivity analysis described in Sect. 5.2.

- Finally it has to be mentioned that the test plan had to fit in the soundboard and piano production scheduling, which is not a continuous one, but includes prolonged seasoning phases.

Moreover, additional experimental data not used for material parameter identification (static deflections after ribs gluing, see Sect.4.2.1 and [22]) were available for validation of the FE model at STAGE 1 , and therefore the experimental characterisation of STAGE 0 was considered not strictly necessary. 


\subsection{Modal assurance criterion and experimental to numerical mode pairing}

The first task to be solved is to pair numerical mode shapes and experimental ones, so as to be able to compare the corresponding natural frequencies. Both the experimental modes and the numerical ones are known at discrete locations: accelerometer positions on the measurement grid and nodes in the FE model. FE nodes are far more numerous than measurement locations: special care was given in order to position a FE node at each position of the measurement grid, in order to allow for direct comparison. Thanks to this, both numerical and experimental modes can be characterised by a vector of displacement components $\mathbf{u}$, where the FE vector is just extracted from the eigenvector pertaining to the whole model.

In order to pair numerical and experimental modes the modal assurance criterion (MAC) was adopted [42]. In general, for complex valued vectors $\mathbf{u}$ and $\mathbf{v}$, the MAC value is defined as

$$
\operatorname{MAC}(\mathbf{u}, \mathbf{v})=\frac{\left|\mathbf{u}^{\mathrm{H}} \mathbf{v}\right|^{2}}{\|\mathbf{u}\|^{2}\|\mathbf{v}\|^{2}}
$$

where $\mathbf{u}^{\mathrm{H}}$ is the conjugate transpose of $\mathbf{u},|z|^{2}=\bar{z} z$ is the squared modulus of the complex number $z$, and $\|\mathbf{u}\|^{2}=\mathbf{u}^{\mathrm{H}} \mathbf{u}$ is the squared $\ell^{2}$-norm of $\mathbf{u}$. It can be shown that $0 \leq \operatorname{MAC}(\mathbf{u}, \mathbf{v}) \leq 1$, where MAC $=1$ is attained when vectors $\mathbf{u}$ and $\mathbf{v}$ are identical up to a complex modal scaling factor, while MAC $=0$ denotes completely uncorrelated modal vectors.

The general MAC criterion can also be used in the present case, where the eigenmodes are real valued: in (5) no damping was introduced, while the identified experimental modes are assumed to be real-valued under the hypothesis of lightly damped system. Denoting by $\left(f_{\mathrm{x}}^{(r)}, \mathbf{u}_{\mathrm{x}}^{(r)}\right), r=1 \ldots P$ the set of experimentally identified natural frequencies and mode shapes and by $\left(f_{\mathrm{n}}^{(s)}, \mathbf{u}_{\mathrm{n}}^{(s)}\right), s=1 \ldots Q$ the numerical ones, a MAC-matrix $\mathbf{M}$ with elements

$$
M_{r s}=\operatorname{MAC}\left(\mathbf{u}_{\mathrm{x}}^{(r)}, \mathbf{u}_{\mathrm{n}}^{(s)}\right)
$$

can be defined $5^{5}$ Two modes $\left(f_{\mathrm{x}}^{(r)}, \mathbf{u}_{\mathrm{x}}^{(r)}\right)$ and $\left(f_{\mathrm{n}}^{(s)}, \mathbf{u}_{\mathrm{n}}^{(s)}\right)$ are paired if $M_{r s}>m_{\mathrm{t}}$, where $m_{\mathrm{t}}$ is a suitably defined threshold. If $N_{\text {pair }}$ is the number of paired modes, this procedure defines a mapping $\left(r_{i}, s_{i}\right), i=1 \ldots N_{\text {pair }}$, between the paired experimental and numerical mode indexes $r, s$.

\subsection{Material parameters updating at STAGE 1}

At STAGE 1 the soundboard is made of Norway spruce (board) and Sitka spruce (ribs), the elastic properties of which are described by 10 parameters each, see Tab. 2. First of all a Design Sensitivity Analysis (DSA) was performed on the first 40 modes of the soundboard, calculating the change in natural frequency caused by small changes in each elastic constant. This procedure showed that only a limited number of elastic properties needed to be taken into account for model updating. The most important were found to be $E_{1}$ of both Norway and Sitka spruce.

\footnotetext{
${ }^{5} \mathrm{~A}$ colour scale representation of these MAC-matrices is given in fig. 7
}

DSA demonstrated also that $E_{2}$ of Norway spruce has an effect on the natural frequency smaller than $E_{1}$ of Sitka spruce as the ribs tend to dominate the stiffness in this direction. Changes in $G_{13}$ of Sitka spruce affect natural frequencies only below the tenth mode. The other moduli and Poisson ratios have negligible impact and were not included in the updating procedure.

As a consequence of DSA and considering uncertainties in the density and overall stiffness of the wood, the following six parameters were selected for independent updating:

- a common scaling factor for all the elastic moduli of both woods;

- a scaling factor for all the elastic moduli of Sitka Spruce alone;

- $E_{1}$ of Sitka spruce;

- $E_{1}$ of Norway spruce;

- ratio $E_{1} / G_{13}$ for Sitka spruce;

- density of Sitka spruce (density of Norway spruce is consequently updated to match the soundboard mass measured at STAGE 1 during modal testing).

The purpose of the material parameters updating is to obtain a better match between the numerical simulation and the experimental data. If one has $N_{\text {pair }}$ paired modes $\left(r_{i}, s_{i}\right), i=1 \ldots N_{\text {pair }}$, the objective is to minimise

$$
g=\sum_{i=1}^{N_{\text {pair }}} \alpha^{2}\left[1-\frac{f_{\mathrm{n}}^{\left(s_{i}\right)}}{f_{\mathrm{x}}^{\left(r_{i}\right)}}\right]^{2}+\beta^{2}\left[1-M_{r_{i} s_{i}}\right]^{2}
$$

i. e. to have better natural frequency and mode shape matching. The evaluation of $g$ requires the solution of a multi-step nonlinear FE analysis, and obviously has a highly nonlinear dependence on the material parameters under investigation.

A solution to the above defined minimisation problem was pursued by coupling the MATLAB non linear least square solver lsqnonlin and Abaqus itself. No claim is made about having computed a true optimal solution in this way. In fact special care was dedicated in finding physically admissible values for the material parameters: therefore somewhat arbitrary but realistic bounds were imposed on the material parameters in the least squares solving process.

After numerous experiments, the updated parameters presented in Tab. 2 were considered acceptable for the purpose of the present investigation. One can note that the changes in the material parameters recorded in Tab. 2 are well below the typical coefficient of variation of measured wood mechanical properties (see e. g. Tab. 4-6 in [39]).

As it is detailed in the next section, the agreement between numerical and experimental modes is still very good at STAGE 2, after bridge gluing: therefore a more detailed analysis of the bridge material parameters was considered purposeless. 

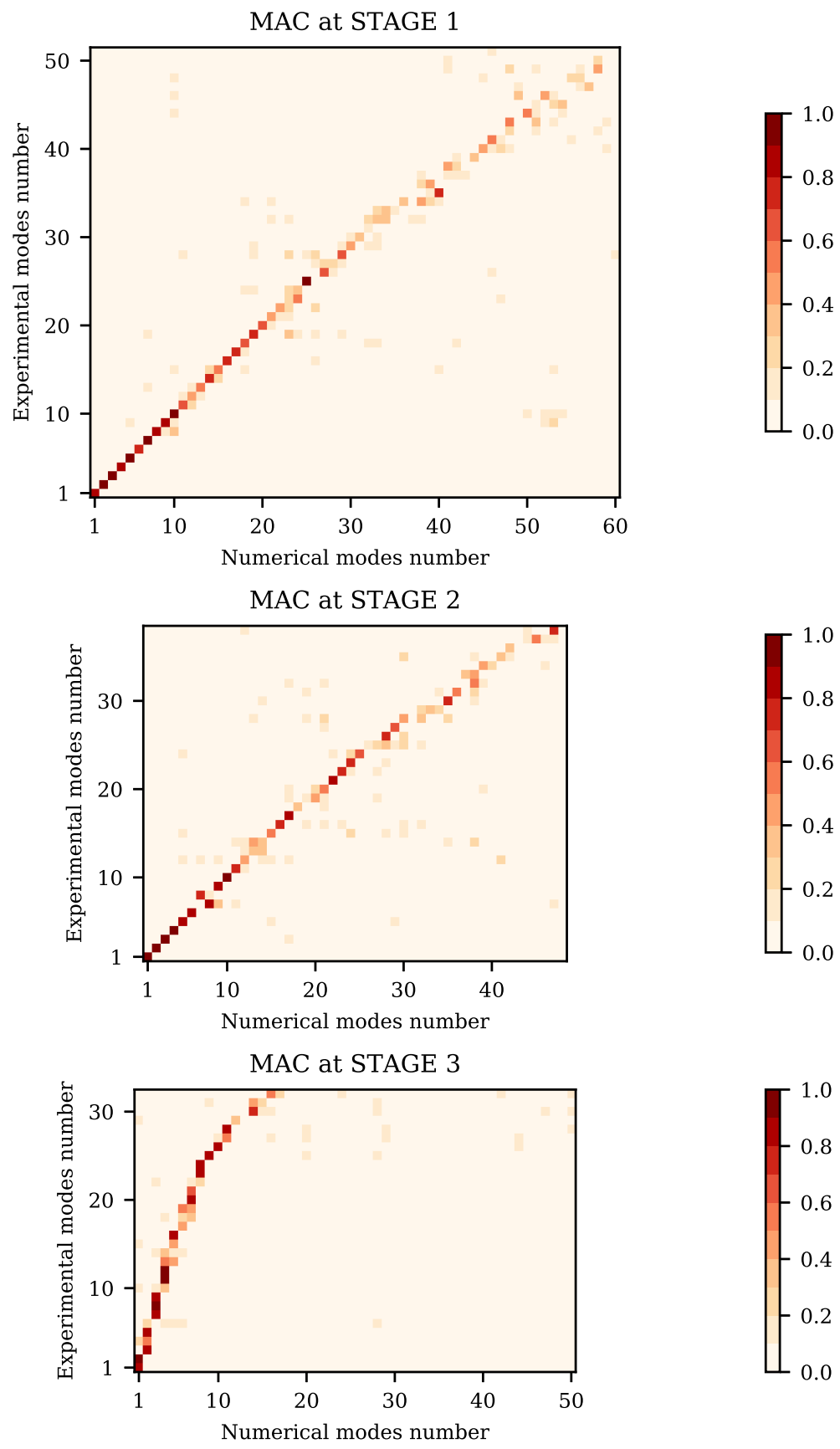

Figure 7: Colour scale representation of the numerical to experimental MAC matrix, after material parameter updating. 


\section{Numerical-experimental comparison}

The comparison between numerical and experimental modes is presented in terms of the MAC matrix in Fig. 7, while Figs. 8 to 11 show the comparison between the paired numerical and experimental modes, in terms of natural frequency, MAC value, and visual representation of the mode shapes. The comparison is repeated for the first 6 modes of stages 1 and 2 (Figs. 8. 9) and the first 12 experimental modes for stage 3 (Figs. 10 11). In Tab. 3 the comparison is extended to the first 20 experimental modes. All the numerical results correspond to the updated material parameters, obtained from the procedure described in the previous section.

Given that in the experiments of stages 1 and 2 the soundboard was suspended on a steel cable, the tested condition is assumed to correspond to the free-edge boundary condition applied in the FE simulations. In fact a one-to-one correspondence between numerical and experimental modes is found, with the only exception of a limited number of experimental modes that do not have a FE counterpart.

In the case of STAGE 3 the situation is different. While the tested structure is the soundboard assembled in the piano case (resting on three supports located under the rim), the FE one is the soundboard alone, clamped all along its edge. The latter modelling choice corresponds to assuming the rim to be infinitely rigid. On the contrary, experimental modal analysis at STAGE 3 revealed that the soundboard-rim structural coupling results in vibration mode duplications (twin or family modes), i. e. the same mode computed through the clamped-edge FE model may correspond to more than one experimental mode with very similar shape but different natural frequency (see also [6, 7, 21]). This circumstance is evidenced through the way numerical and experimental modes of stAGE 3 are associated in Tab. 3. Note that the clamped-edge assumption of the FE model leads to a systematic overestimation of the natural frequencies.

The results reported in Tab. 3 refer to the low frequency range, i. e. the range where experimental modal analysis can be successfully performed and single vibration modes identified. Actually, it is well known that the FRF of a plate-like structure is dominated by individual resonances in the low-frequency range, while at higher frequency the increasing modal density makes it impossible to separate the different modes. In the specific case analysed in this paper, the transition frequency between these two kind of response varies with the manufacturing stage, as evidenced in Sect. 3. For each stage, the validation of the FE model in terms of single vibration modes can be carried out up to this transition frequency at most. Although experimental modal analysis allowed identifying modes up to $400 \mathrm{~Hz}-450 \mathrm{~Hz}$, for the sake of brevity the numerical-experimental comparison reported in Tab. 3 is limited to the first 20 experimental modes of each manufacturing stage.

However, together with mode pairing it is interesting to verify if the model is capable of reproducing the soundboard dynamic response over a wider frequency range. To this end the FE model was used for calculating the FRF up to $4 \mathrm{kHz}$. Fig. 12 shows the comparison between calculated and experimental point mobility: the soundboard is excited in the same position considered in Fig. 5 (i.e. on to the bridge, in correspondence with note G3). The numerical calculation is performed by modal superposition and the damping ratios $\xi_{n}$ are those obtained from modal testing, where available (see Tab. 1), and set to $1 \%$ for all modes at higher frequency. This corresponds to a loss factor $\eta_{n}=2 \xi_{n}$ of $2 \%$ and is aligned with the values found in [19] where the average loss factor, up to $3 \mathrm{kHz}$, was $2 \%$, with a range $1 \%$ to $3 \%$.

The agreement between modelling and measurement is generally good. Below $200 \mathrm{~Hz}$ the model is stiffer and overestimates the natural frequencies. In this range there are three predominant modes in the FE calculation while there are about ten which can be distinguished in the measured mobility curve. This is due to the fact that the FE model does not include the rim and that the soundboard support is assumed to be infinitely rigid. The modes resulting from the coupling with the supporting structure cannot be reproduced by the numerical simulation and the clamped boundary condition makes the model stiffer than the real soundboard. The model predicts correctly the antiresonance at $200 \mathrm{~Hz}$. At higher frequency the effect of the boundary conditions becomes less and less important and the agreement between calculated and measured mobility is very good. The increase in point mobility found in measurements at about $2.5 \mathrm{kHz}$ is also predicted by the FE model but seems to take place at higher frequencies.

\section{Conclusions}

The results obtained from a comprehensive modal analysis campaign were used to validate and update the finite element model of a grand piano soundboard. Considering the characteristics of the soundboard structure and of its manufacturing process, it was decided to develop the finite element model of a structure with increasing complexity, by following the same progressive stages adopted in the instrument construction. Therefore, testing and simulation proceeded in parallel, so as to improve the model capability of capturing the dynamic behaviour of the real structure, which depends on the combination of many parameters, ranging from material properties to geometric configuration, from process-induced stresses to boundary conditions.

Bearing in mind the unavoidable uncertainties which are typical of a complex natural material like wood, at STAGE 1 the soundboard material properties were updated so as to minimise the difference between calculated and experimental vibration modes, in terms of both natural frequencies and mode shapes. Any other model feature was left unvaried. Then the FE simulation of the manufacturing process proceeded in STAGE 2 and 3, without any further tuning of the material properties and, at each stage, the agreement between numerical results and experimental data was verified.

Experimental modal analysis allowed identifying the vibration modes in the low-frequency range only, where the soundboard dynamic response is dominated by individual modal resonances. In the specific case of the tested piano soundboard, this corresponds to approximately 400 to $450 \mathrm{~Hz}$ maximum. Accordingly, model validation in terms of numerical-experimental comparison of single natural frequencies and corresponding 


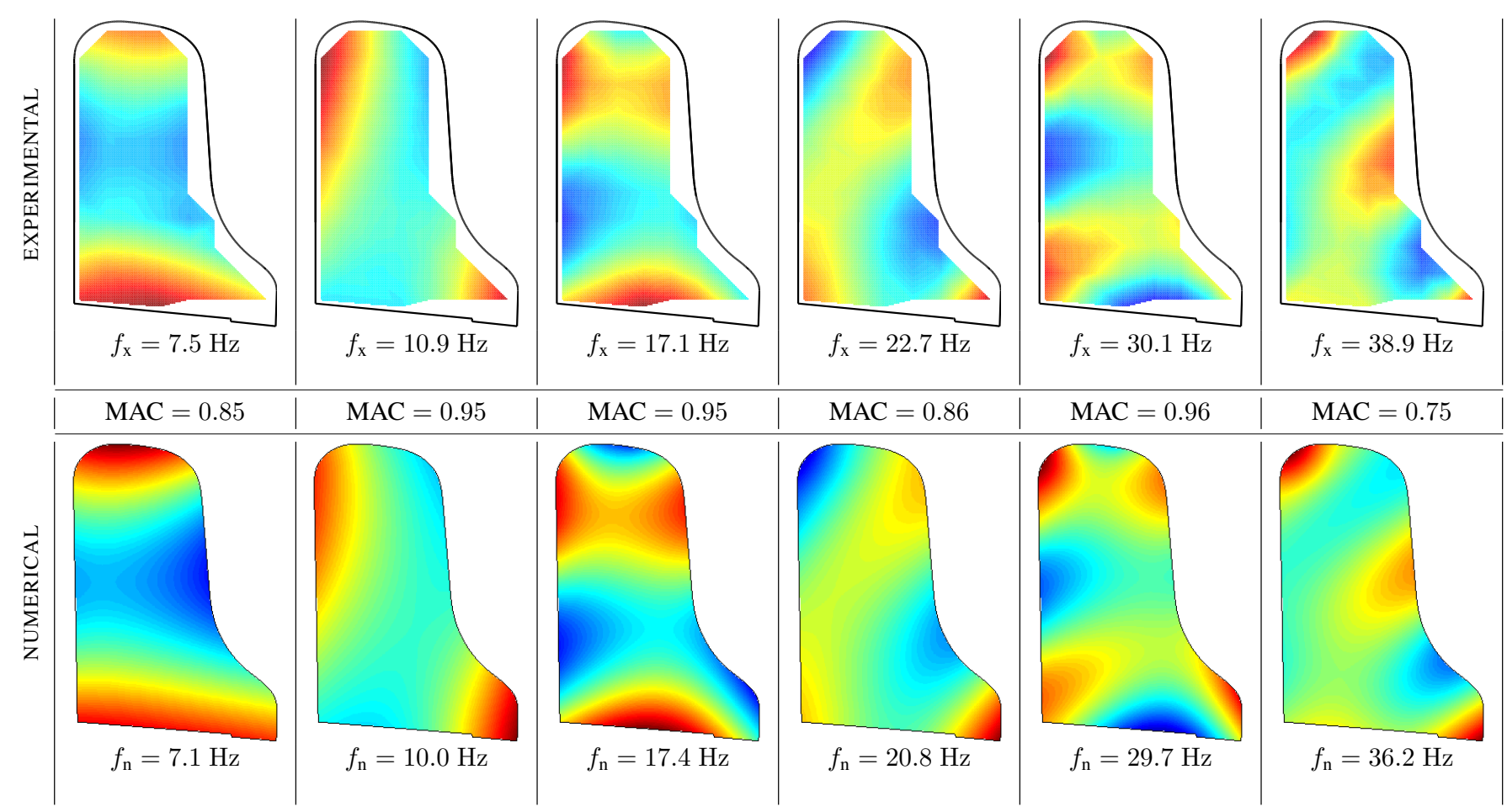

Figure 8: STAGE 1: first 6 experimental vibration modes vs. numerical ones.
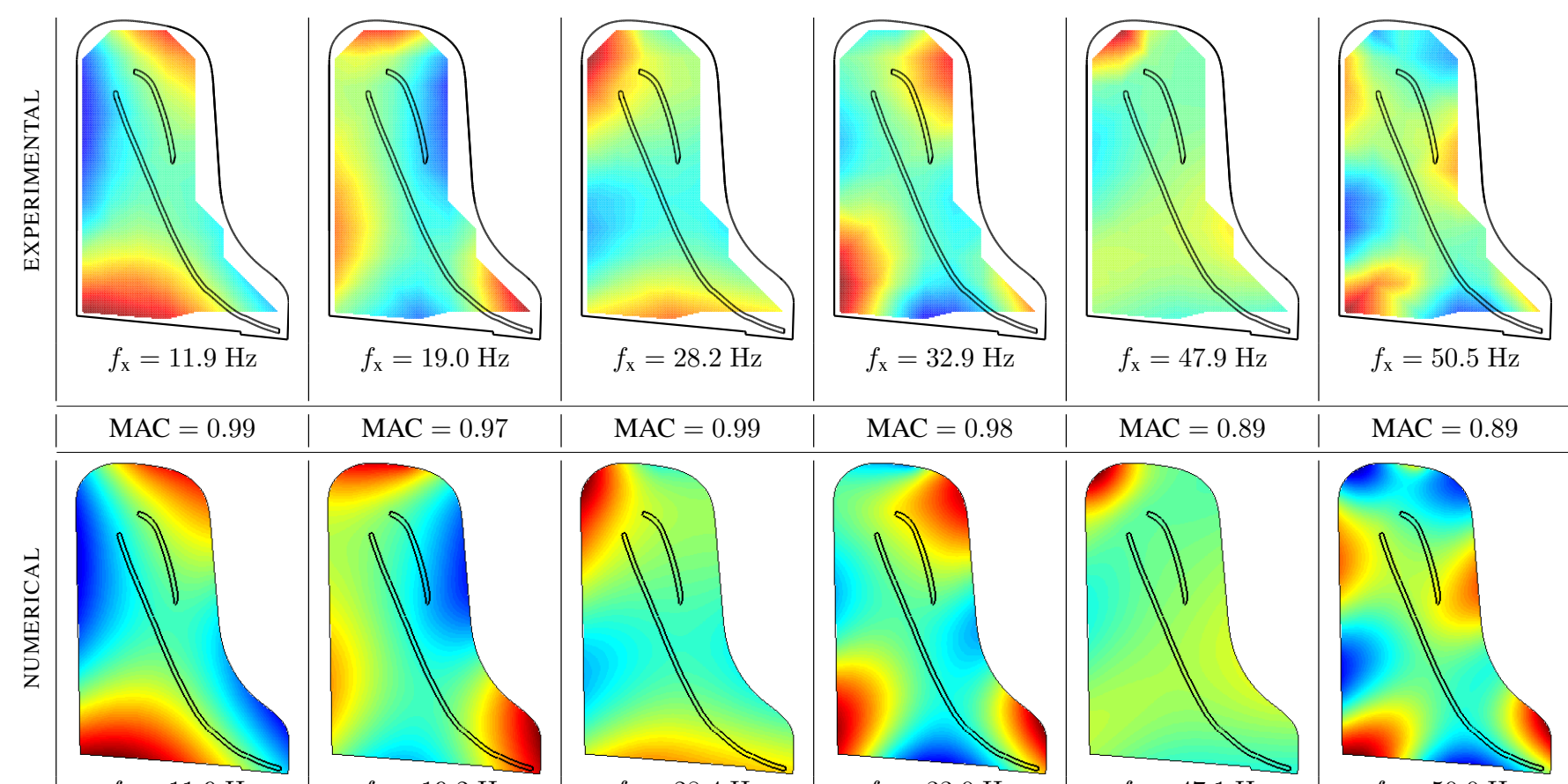

$f_{\mathrm{n}}=11.9 \mathrm{~Hz}$
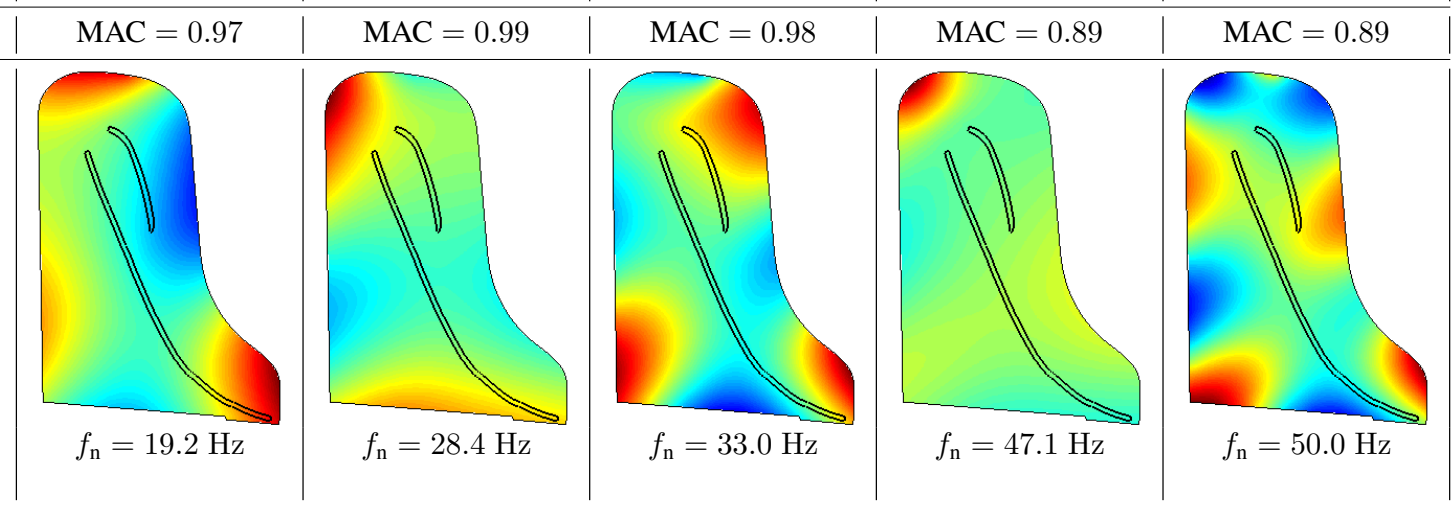

Figure 9: STAGE 2: first 6 experimental vibration modes vs. numerical ones. 

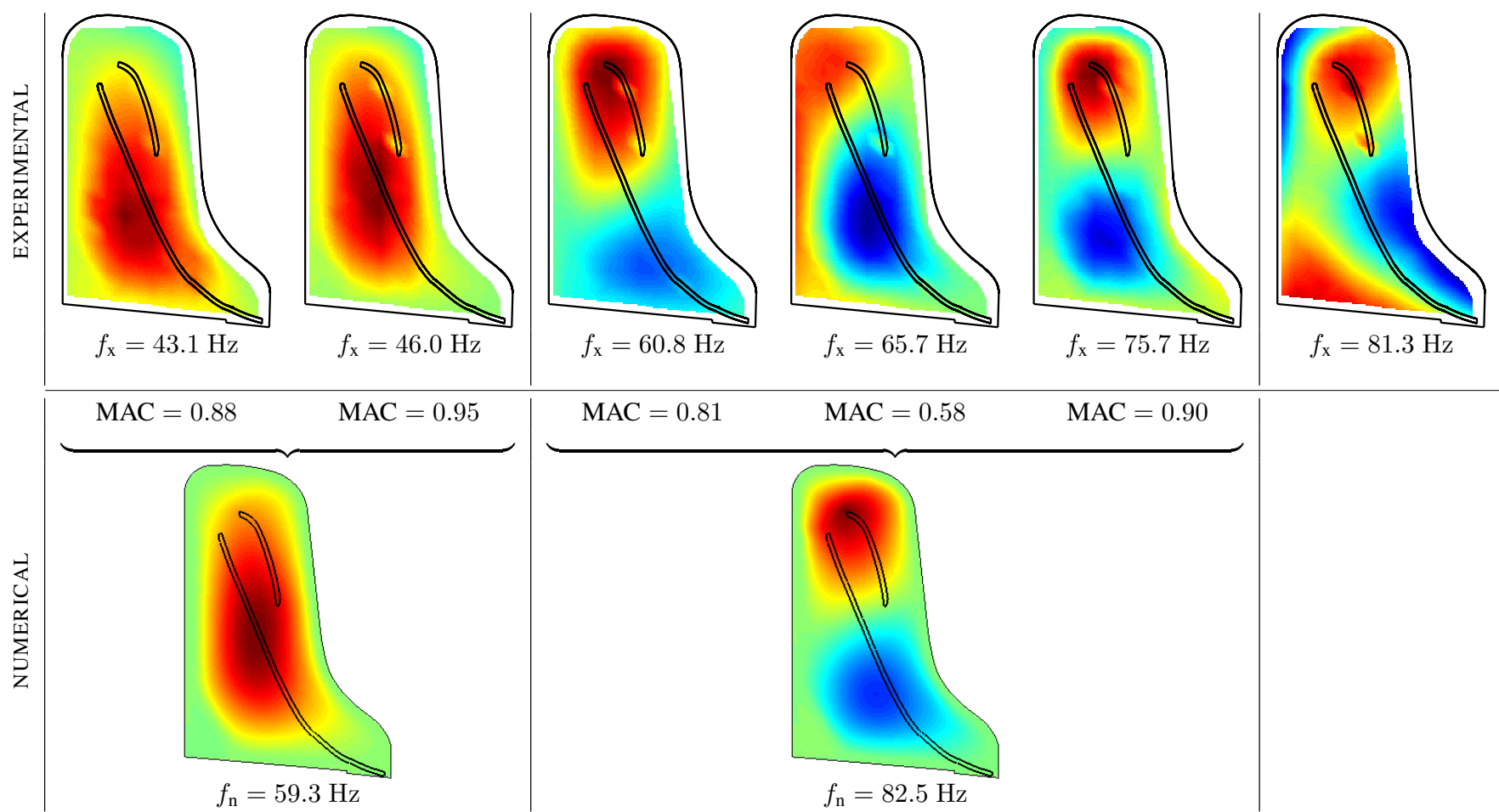

Figure 10: staGe 3: first 6 experimental vibration modes vs. numerical ones.

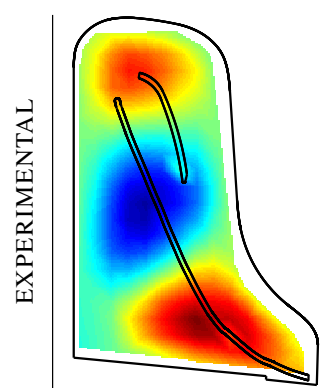

$f_{\mathrm{x}}=93.5 \mathrm{~Hz}$

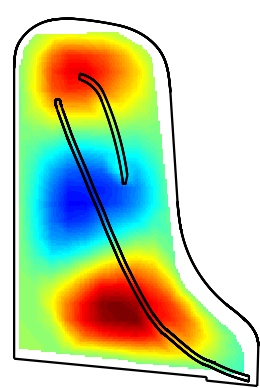

$f_{\mathrm{x}}=103.9 \mathrm{~Hz}$

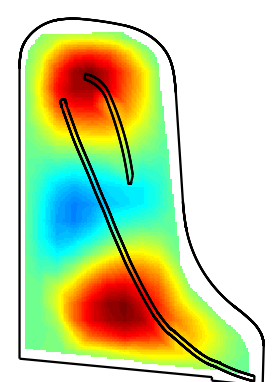

$f_{\mathrm{x}}=112.9 \mathrm{~Hz}$

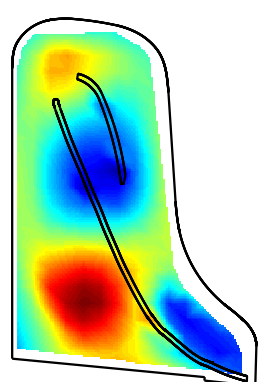

$f_{\mathrm{x}}=126.3 \mathrm{~Hz}$

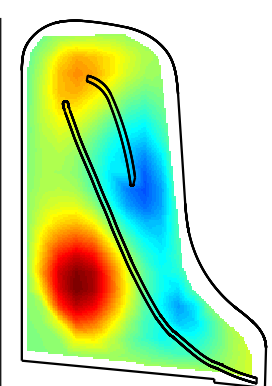

$f_{\mathrm{x}}=135.9 \mathrm{~Hz}$

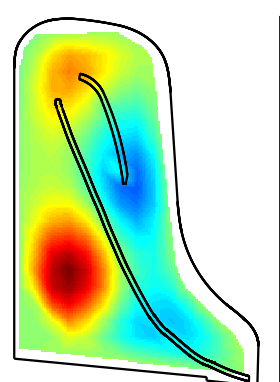

$f_{\mathrm{x}}=142.3 \mathrm{~Hz}$
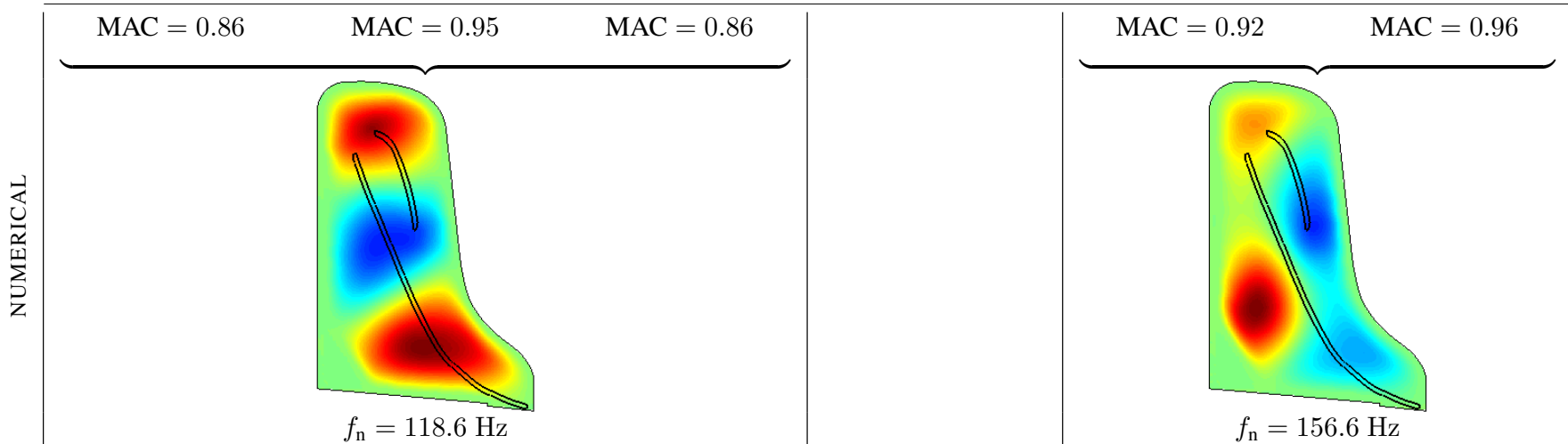

Figure 11: sTAGE 3: second 6 experimental vibration modes vs. numerical ones. 
Table 3: Experimental vs. numerical vibration modes.

\begin{tabular}{|c|c|c|c|c|c|c|c|c|}
\hline \multicolumn{3}{|c|}{ STAGE 1} & \multicolumn{3}{|c|}{ STAGE 2} & \multicolumn{3}{|c|}{ STAGE 3} \\
\hline $\begin{array}{l}\text { nat. freq. of the } \\
\text { experimental } \\
\text { modes }(\mathrm{Hz})\end{array}$ & MAC & $\begin{array}{c}\text { nat. freq. of the } \\
\text { numerical } \\
\text { modes }(\mathrm{Hz})\end{array}$ & $\begin{array}{c}\text { nat. freq. of the } \\
\text { experimental } \\
\text { modes }(\mathrm{Hz})\end{array}$ & MAC & $\begin{array}{c}\text { nat. freq. of the } \\
\text { numerical } \\
\text { modes }(\mathrm{Hz})\end{array}$ & $\begin{array}{c}\text { nat. freq. of the } \\
\text { experimental } \\
\text { modes }(\mathrm{Hz})\end{array}$ & MAC & $\begin{array}{l}\text { nat. freq. of the } \\
\text { numerical } \\
\text { modes }(\mathrm{Hz})\end{array}$ \\
\hline 7.5 & 0.85 & 7.1 & 11.9 & 0.99 & 11.9 & 43.1 & 0.88 & 593 \\
\hline 10.9 & 0.95 & 10.0 & 19.0 & 0.97 & 19.2 & 46.0 & 0.95 & 59.3 \\
\hline 17.1 & 0.95 & 17.4 & 28.2 & 0.99 & 28.4 & 60.8 & 0.81 & \\
\hline 22.7 & 0.86 & 20.8 & 32.9 & 0.98 & 33.0 & 65.7 & 0.58 & 82.5 \\
\hline 30.1 & 0.96 & 29.7 & 47.9 & 0.89 & 47.1 & 75.7 & 0.90 & \\
\hline 38.9 & 0.75 & 36.2 & 50.5 & 0.89 & 50.0 & 81.3 & & \\
\hline 43.8 & 0.95 & 43.6 & 61.1 & 0.87 & 65.5 & 93.5 & 0.86 & \\
\hline 55.7 & 0.85 & 53.1 & 63.3 & 0.75 & 64.7 & 103.9 & 0.95 & 118.6 \\
\hline 56.7 & 0.87 & 57.2 & 70.9 & 0.80 & 74.5 & 112.9 & 0.86 & \\
\hline 65.9 & 0.94 & 65.4 & 77.7 & 0.91 & 80.4 & 126.3 & & \\
\hline 74.3 & 0.64 & 74.1 & 85.6 & 0.78 & 89.5 & 135.9 & 0.92 & \\
\hline 75.8 & & & 89.9 & & & 142.3 & 0.96 & 156.6 \\
\hline 80.5 & 0.58 & 81.2 & 100.7 & & & 151.5 & & \\
\hline 92.1 & 0.71 & 92.6 & 105.8 & 0.49 & 105.9 & 153.6 & & \\
\hline 96.9 & 0.58 & 97.7 & 111.4 & 0.54 & 115.7 & 157.1 & & \\
\hline 102.4 & 0.74 & 104.5 & 125.9 & 0.72 & 128.1 & 161.5 & 0.86 & 175.9 \\
\hline 111.2 & 0.73 & 112.7 & 131.4 & 0.85 & 133.7 & 170.9 & 0.47 & 198.0 \\
\hline 119.1 & 0.63 & 115.2 & 139.1 & & & 174.8 & & \\
\hline 127.5 & 0.71 & 128.7 & 153.3 & 0.45 & 157.7 & 185.0 & & \\
\hline 134.8 & 0.69 & 136.3 & 167.1 & 0.54 & 170.6 & 191.2 & 0.81 & 204.8 \\
\hline
\end{tabular}

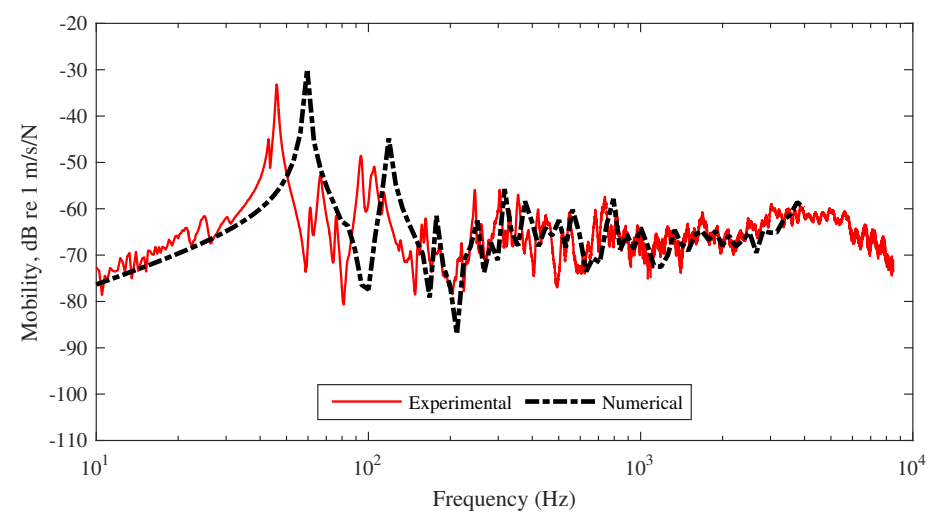

Figure 12: STAGE 3: experimental vs. numerical point mobilities evaluated on the bridge at note G3. 
mode shapes could be performed only in this frequency range. The number of experimental modes which are involved in this validation process ranges from 34 to 52, depending on the manufacturing stage. Once that the FE model was properly tuned in terms of individual vibration modes, numerical and experimental FRF's were compared, to verify the model capability of reproducing the structure's dynamic behaviour also in the $\mathrm{mid} /$ high-frequency range, which is characterised by increasing modal density and coupled multi-mode response. The point mobility measured on the bridge at STAGE 3 was compared with the corresponding numerical result; the two are good agreement in the range $200 \mathrm{~Hz}$ to $4000 \mathrm{~Hz}$. Below $200 \mathrm{~Hz}$ the main differences are due to the flexibility of the rim, this was not included in the model but only represented by clamped boundary conditions. This effect becomes negligible above $200 \mathrm{~Hz}$.

The results obtained are encouraging and the FE model that has been set up can be considered the first fundamental step towards the development of a vibroacoustic model of the piano soundboard. The main advantage of the modelling approach proposed in this paper is that it can provide a better understanding of the effect of the manufacturing process and of specific design solutions on the soundboard vibration properties and on its consequent acoustic performance.

\section{References}

[1] P. H. Bilhuber, C. A. Johnson, The Influence of the Soundboard on Piano Tone Quality, The Journal of the Acoustical Society of America 11 (3) (1940) 311-320. doi:10.1121/1.1916039

[2] K. Wogram, Acoustical research on pianos: vibrational characteristics of the soundboard, Das Musikinstrument 24 (1980) 694-702.

[3] K. Wogram, The strings and the soundboard, in: A. Askenfelt (Ed.), Five Lectures on the Acoustics of pianos, 1990.

[4] N. Giordano, Mechanical impedance of a piano soundboard, The Journal of the Acoustical Society of America 103 (4) (1998) 2128-2133. doi : $10.1121 / 1.421358$

[5] I. Nakamura, The vibrational character of the piano soundboard, in: Proceedings of the 11th ICA, Vol. 4, 1983, pp. 385-388.

[6] H. Suzuki, Vibration and sound radiation of a piano soundboard, The Journal of the Acoustical Society of America 80 (6) (1986) 1573-1582. doi:10.1121/1.394321

[7] J. Kindel, I.-C. Wang, Modal Analysis and finite element analysis of a piano soundboard, in: 5th International Modal Analysis Conference (IMAC), 1987, pp. 1545-1549.

[8] H. A. Conklin, Design and tone in the mechanoacoustic piano. Part II. Piano structure, The Journal of the Acoustical Society of America 100 (2) (1996) 695-708. doi:10.1121/1.416233

[9] N. Giordano, A. J. Korty, Motion of a piano string: Longitudinal vibrations and the role of the bridge, The Journal of the Acoustical Society of America 100 (6) (1996) 3899-3908. doi:10.1121/1.417219

[10] N. Giordano, Simple model of a piano soundboard, The Journal of the Acoustical Society of America 102 (2) (1997) 1159-1168. doi:10.1121/ 1.419868

[11] N. Giordano, Sound production by a vibrating piano soundboard: Experiment, The Journal of the Acoustical Society of America 104 (3) (1998) 1648-1653. doi:10.1121/1.424377

[12] N. Giordano, M. Jiang, Physical modeling of the piano, EURASIP Journal on Applied Signal Processing 2004 (7) (2004) 926-933. doi:10.1155/ S111086570440105X

[13] J. Chabassier, A. Chaigne, P. Joly, Modeling and simulation of a grand piano, The Journal of the Acoustical Society of America 134 (1) (2013) 648-665. doi:10.1121/1.4809649

[14] J. Berthaut, M. N. Ichchou, L. Jézéquel, Piano soundboard: structural behavior, numerical and experimental study in the modal range, Applied
Acoustics 64 (11) (2003) 1113-1136. doi:10.1016/S0003-682X (03) 00065-3

[15] T. R. Moore, S. A. Zietlow, Interferometric studies of a piano soundboard, The Journal of the Acoustical Society of America 119 (3) (2006) 17831793. doi:10.1121/1.2164989

[16] A. Mamou-Mani, J. Frelat, C. Besnainou, Numerical simulation of a piano soundboard under downbearing, The Journal of the Acoustical Society of America 123 (4) (2008) 2401-2406. doi:10.1121/1.2836787

[17] A. Mamou-Mani, S. Le Moyne, F. Ollivier, C. Besnainou, J. Frelat, Prestress effects on the eigenfrequencies of the soundboards: Experimental results on a simplified string instrument, The Journal of the Acoustical Society of America 131 (1) (2012) 872-877. doi:10.1121/1.3651232

[18] A. Chaigne, B. Cotté, R. Viggiano, Dynamical properties of piano soundboards, The Journal of the Acoustical Society of America 133 (4) (2013) 2456-2466. doi:10.1121/1.4794387

[19] K. Ege, X. Boutillon, M. Rébillat, Vibroacoustics of the piano soundboard: (Non)linearity and modal properties in the low- and mid-frequency ranges, Journal of Sound and Vibration 332 (5) (2013) 1288-1305. doi:10, $1016 / j \cdot j$ sv.2012.10.012

[20] X. Boutillon, K. Ege, Vibroacoustics of the piano soundboard: Reduced models, mobility synthesis, and acoustical radiation regime, Journal of Sound and Vibration 332 (18) (2013) 4261-4279. doi : 10.1016/j · jsv 2013.03.015

[21] K. Ege, X. Boutillon, B. David, High-resolution modal analysis, Journal of Sound and Vibration 325 (4-5) (2009) 852-869. doi:10.1016/j j jsv 2009.04.019

[22] R. Corradi, P. Fazioli, S. Miccoli, F. Ripamonti, G. Squicciarini, Experimental modal analysis and structural modelling of a grand piano soundboard to support instrument design, in: Tenth International Conference on Recent Advances in Structural Dynamics (RASD 2010), Institute of Sound and Vibration Research, 2010.

[23] R. Corradi, P. Fazioli, S. Marforio, A. Paluello, S. Miccoli, G. Squicciarini, Modal analysis of a grand piano soundboard, in: International conference on noise and vibration engineering (ISMA 2010), 2010.

[24] K. Fenner, Resonanzboden in Neubau und Reparatur, EuroPiano (1) (1998) $14+$

[25] D. J. Ewins, Modal testing : theory, practice, and application, Research Studies Press, 2000.

[26] J. S. Bendat, A. G. Piersol, Random data : analysis and measurement procedures, Wiley, 2000

[27] W. Heylen, S. Lammens, P. Sas, Modal analysis theory and testing, Katholieke Universiteit Leuven, Faculty of Engineering, Dept. of Mechanical Engineering, Division of Production Engineering, Machine Design and Automation, 1998.

[28] N. M. Maia, J. M. Montalvão e Silva, Theoretical and experimental modal analysis, Research Studies Press, 1997

[29] H. Van der Auweraer, P. Guillaume, P. Verboven, S. Vanlanduit, Application of a Fast-Stabilizing Frequency Domain Parameter Estimation Method, Journal of Dynamic Systems, Measurement, and Control 123 (4) (2001) 651-658. doi:10.1115/1.1410369

[30] E. Skudrzyk, The mean-value method of predicting the dynamic response of complex vibrators, The Journal of the Acoustical Society of America 67 (4) (1980) 1105-1135. doi:10.1121/1.384169

[31] J. Woodhouse, On the synthesis of guitar plucks, Acta Acustica united with Acustica 90 (5) (2004) 928-944.

[32] J. Woodhouse, On the "bridge hill" of the violin, Acta Acustica united with Acustica 91 (1) (2005) 155-165.

[33] B. Elie, F. Gautier, B. David, Macro parameters describing the mechanical behavior of classical guitars, Journal of the Acoustical Society of America 132 (6) (2012) 4013-4024. doi:10.1121/1.4765077

[34] B. Elie, F. Gautier, B. David, Estimation of mechanical properties of panels based on modal density and mean mobility measurements, Mechanical Systems and Signal Processing 40 (2) (2013) 628-644. doi:10.1016/j ymssp.2013.06.009

[35] K. Ege, X. Boutillon, Global and local synthetic descriptions of the piano soundboard, in: Proceedings of Forum Acusticum, 2011, pp. 473-478.

[36] X. Boutillon, K. Ege, Vibroacoustics of the piano soundboard: Reduced models, mobility synthesis, and acoustical radiation regime, Journal of Sound and Vibration 332 (18) (2013) 4261-4279. doi : 10.1016/j · j sv 2013.03.015

[37] Abaqus User Manual (2010). 
[38] V. Bucur, Acoustics of wood, Springer, 2006.

[39] D. W. Green, J. E. Winandy, D. E. Kretschmann, Mechanical Properties of Wood, Gen. Tech. Rep. FPL-GTR-113, Forest Products Laboratory USDA Forest Service Madison, Wisconsin, 1999, Ch. 4.

[40] K.-J. Bathe, Finite element procedures, Klaus-Jürgen Bathe, Watertown, 2014.

[41] O. C. Zienkiewicz, R. L. Taylor, The Finite Element Method, Vol. 2: Solid Mechanics, Butterworth Heineman, 2000.

[42] R. J. Allemang, D. L. Brown, Correlation coefficient for modal vector analysis, in: Proceedings of the International Modal Analysis Conference \& Exhibit, 1982, pp. 110-116.

\section{A. Naming convention for orthotropic elastic constants}

The engineering constants $E_{i}, v_{i j}, G_{i j}$ reported in Tab. 2 are defined in terms of elastic compliance as follows:

$$
\left\{\begin{array}{c}
\varepsilon_{1} \\
\varepsilon_{2} \\
\varepsilon_{3} \\
\gamma_{23} \\
\gamma_{31} \\
\gamma_{12}
\end{array}\right\}=\left[\begin{array}{cccccc}
\frac{1}{E_{1}} & -\frac{v_{21}}{E_{2}} & -\frac{v_{31}}{E_{3}} & 0 & 0 & 0 \\
-\frac{v_{12}}{E_{1}} & \frac{1}{E_{2}} & -\frac{v_{32}}{E_{3}} & 0 & 0 & 0 \\
-\frac{v_{13}}{E_{1}} & -\frac{v_{23}}{E_{2}} & \frac{1}{E_{3}} & 0 & 0 & 0 \\
0 & 0 & 0 & \frac{1}{G_{23}} & 0 & 0 \\
0 & 0 & 0 & 0 & \frac{1}{G_{31}} & 0 \\
0 & 0 & 0 & 0 & 0 & \frac{1}{G_{12}}
\end{array}\right]\left\{\begin{array}{c}
\sigma_{1} \\
\sigma_{2} \\
\sigma_{3} \\
\tau_{23} \\
\tau_{31} \\
\tau_{12}
\end{array}\right\}
$$

with

$$
-\frac{v_{21}}{E_{2}}=-\frac{v_{12}}{E_{1}}, \quad-\frac{v_{31}}{E_{3}}=-\frac{v_{13}}{E_{1}}, \quad-\frac{v_{32}}{E_{3}}=-\frac{v_{23}}{E_{2}},
$$

for a total of 9 independent elastic constants. The elasticity axes $(1,2,3)$ are aligned to the anatomical directions $(\mathrm{L}, \mathrm{R}, \mathrm{T})$, i. e. longitudinal, radial, and tangential to the annual rings.

\section{List of acronyms}

$\begin{array}{ll}\text { CAD } & \text { Computer Aided Design } \\ \text { CNC } & \text { Computer Numerical Control } \\ \text { DSA } & \text { Design Sensitivity Analysis } \\ \text { FE (FEM) } & \text { Finite Element (Method) } \\ \text { FFT } & \text { Fast Fourier Transform } \\ \text { FRF } & \text { Frequency Response Function } \\ \text { IRF } & \text { Impulse Response Function } \\ \text { LSCE } & \text { Least Squares Complex Exponential } \\ \text { MAC } & \text { Modal Assurance Criterion }\end{array}$

